\title{
Multi-source random excitation identification for stochastic structures based on matrix perturbation and modified regularization method
}

\author{
Z.C. $\mathrm{He}^{1}$, Zhuomin Zhang ${ }^{1}$, Eric $\mathrm{Li}^{2 *}$ \\ ${ }^{1}$ State Key Laboratory of Advanced Design and Manufacturing for Vehicle Body, Hunan University, Changsha, \\ 410082 P. R. China \\ ${ }^{2}$ School of Science, Engineering \& Design, Teesside University, Middlesbrough, UK
}

\begin{abstract}
In view of the characteristic of pseudo excitation and effects of the regularization parameter $\lambda$ on the residual norm and solution norm, a novel modified regularization method is proposed to solve the ill-posed problem and mitigate the error propagation of random dynamic loads identification. Compared with Moore-Penrose pseudo inverse and Tikhonov regularization methods that are sensitive to the selection of measurement locations, the proposed modified regularization method can always identify the loads accurately and stably regardless of measurement locations. In addition, the identified loads always match the actual ones from low to high frequency domains using the proposed modified regularization method. Furthermore, the matrix perturbation method is combined with the modified regularization method to analyze the multisource loads acting on the uncertain structure. Several practical engineering examples are conducted to demonstrate that the lower and upper bounds of identified forces can be obtained, which clearly validates the effectiveness and feasibility of the proposed methods in the application of complicated structures.
\end{abstract}

Key words: random dynamic loads identification; stochastic structures; matrix perturbation; modified regularization method

\footnotetext{
* Corresponding author. Tel./fax: +86 73188822051.

E-mail address: ericsg2012@gmail.com
} 


\section{Introduction}

In consideration of evaluating fatigue strength and safety for engineering structures, it is necessary to obtain random dynamic loads exactly, which exist extensively in various practical engineering fields, such as the buffet loads acting on the airframe structure of aircraft, the exciting force from the road imposed on a moving vehicle and the buildings subjected to seismic loads. However, it is generally difficult or even impractical to measure random dynamic loads directly due to the restriction of engineering technology nowadays. On the contrary, it is relatively an easier way to measure the dynamic responses of the structure including dynamic strains, dynamic displacements or accelerations, which can be adopted to identify the dynamic loads in an indirect way [1-3]. With regard to engineering structures subjected to multiple stationary random dynamic loads, the load identification is the so-called multi-input-multi-output problems in most circumstances, utilizing the power spectral density (PSD) of the dynamic responses and structural properties to identify the information of multi-source random dynamic excitations.

The methods of identifying dynamic loads indirectly have been studied and developed comprehensively in last several decades [4-9], mainly including frequency-domain and time-domain load identification methods, namely so-called second type of inverse problem[10, 11]. Early in the 1979, Bartlett [12] used the least-square scheme to predict the vertical and lateral hub forces in an experimental helicopter model. The least-squares method on the basis of frequency response function (FRF) is applied extensively for various load identification problems. Okubo [13] studied the identification of force generated by a 
machine under operating condition and found that the measured response containing noise, even a minor error, can affect badly the accuracy and stability of force identification on account of ill-conditioned FRF matrix at frequencies near the structural resonances. The ill-posed problem in the inversion process seems to be numerically unstable and inevitable [14]. Hence there are various technologies that have been proposed to handle the ill-posed problem and improve the accuracy of the load identification. Callahna [15] proved that the regularization method is feasible and effective for the ill-posed problem based on singular value decomposition (SVD) technique used during the inversion of FRF matrix. Liu et al. [16] applied the enhanced least squares and total least-squares (TLS) schemes in frequency domain to identify dynamic force, considering the measuring noise of responses.

To date, the random dynamic load identifications are mostly studied based on deterministic structures. Monte Carlo simulation (MCS) $[17,18]$ method is generally used to study the problem about stochastic structure, and it has quite strong applicability and feasibility so that it is usually deemed as reference method for checking others. Nonetheless, it has limitation ineluctably such as enormous computational complexity and deficiency of uniform convergence criterion. Besides the MCS method, orthogonal polynomial expansion method, stochastic finite element method and perturbation method are also widely used for combined random responses analysis [19-22]. The perturbation method [23, 24] converts the intricate stochastic system to several straightforward equations based on Taylor series expansion with obvious advantage of less computational cost and simple application in practical circumstance. 
Nevertheless, it must be taken into consideration that the geometrical and physical characteristics of dynamic structures are generally stochastic because of the incomplete controllability of manufacturing as well as measuring precision and the discreteness of structural materials [25-27]. Liu et al. [28, 29] used Gegenbauer polynomial expansion theory and interval analysis method to identify dynamic loads acting on stochastic structures in time domain. He et al. [30] proposed a novel method for load bounds identification for uncertain structures in the frequency domain by applying the Moore-Penrose pseudo-inversion and the truncated total least squares (TTLS). Batou et al. [31-33] used experimental measurements of responses and an uncertain computational model to identify the random loads acting on fuel assemblies. On the basis of Bayesian approach, Zhang and Antoni [34] reconstructed the force and obtained the Bayesian credible intervals, which are built from its posterior probability density function by dealing with model uncertainty and measurement noise. Jia et al. [35, 36] took FRF estimation error and response measuring noise into account for experimental study of random dynamic loads identification based on weighted regularization method and weighted total least squares method, nevertheless, not considering the direct influence of structural random parameters.

In the present study, based on analyzing relative error of identified load, the composite condition number method is applied to select good measurement locations leading to decrease the condition number of FRF matrix. Due to the noise contained in measurement responses, a novel modified regularization method is proposed to solve the ill-posed problem and reduce the error of identified random excitations. Considering the characteristic 
of pseudo excitation and effects of the regularization parameter on the residual norm and solution norm, the modified regularization parameter is formulated for improving the accuracy as well as stability of identified random loads. With regard to random excitation identification for stochastic structures, the IPEM [37] is employed to transform the combined random excitation identification problems into single random problems. On the basis of that, the sensitivities of random excitation with respect to stochastic structural parameters are derived. The perturbation expressions of the structural random excitation by means of a Taylor series expansion are used to convert the problem of random excitation identification for stochastic structures into two kinds of certain inverse problems, namely the random excitation identification on the mean value of structures' random parameters and the sensitivity identification of random excitation to each random parameter. Based on that, the lower and upper bounds, which reflect the statistical characteristic of identified random excitations, are derived. The proposed method can effectively identify the random dynamic loads for stochastic structures with advantages of high computational efficiency and enough precision in circumstance of small random variable coefficient. Compared with Moore-Penrose pseudo inverse method and Tikhonov regularization method, the proposed modified regularization method is demonstrated to be effective and feasible by several numerical simulations. In addition, the numerical examples also validate the effectiveness and applicability of the matrix perturbation method.

The paper is organized as follows: Section 2 briefly describes the forward problem of random dynamic load identification for stochastic structures. In Section 3, a modified 
regularization method is proposed in detail to solve the ill-posed problems. Section 4 introduces the detailed formulation of inverse pseudo excitation perturbation method (IPEPM) for random excitation identification. Finally, several numerical simulations demonstrate the effectiveness and feasibility of the proposed methods in Section 5 and the conclusions are presented in Section 6.

\section{Formulation of forward problem of combined random dynamic load identification}

\subsection{Inverse pseudo excitation method}

With regard to a linear time-invariant deterministic structure subjected to multi-source stationary dynamic loads, the responses PSD matrix can be expressed as follows:

$$
\mathbf{S}_{\mathbf{Y Y}}(\omega)=\mathbf{H}(\omega) \mathbf{S}_{\mathbf{F F}}(\omega) \mathbf{H}(\omega)^{H}
$$

The specific form of Eq. (1) can be illustrated in Fig. 1.

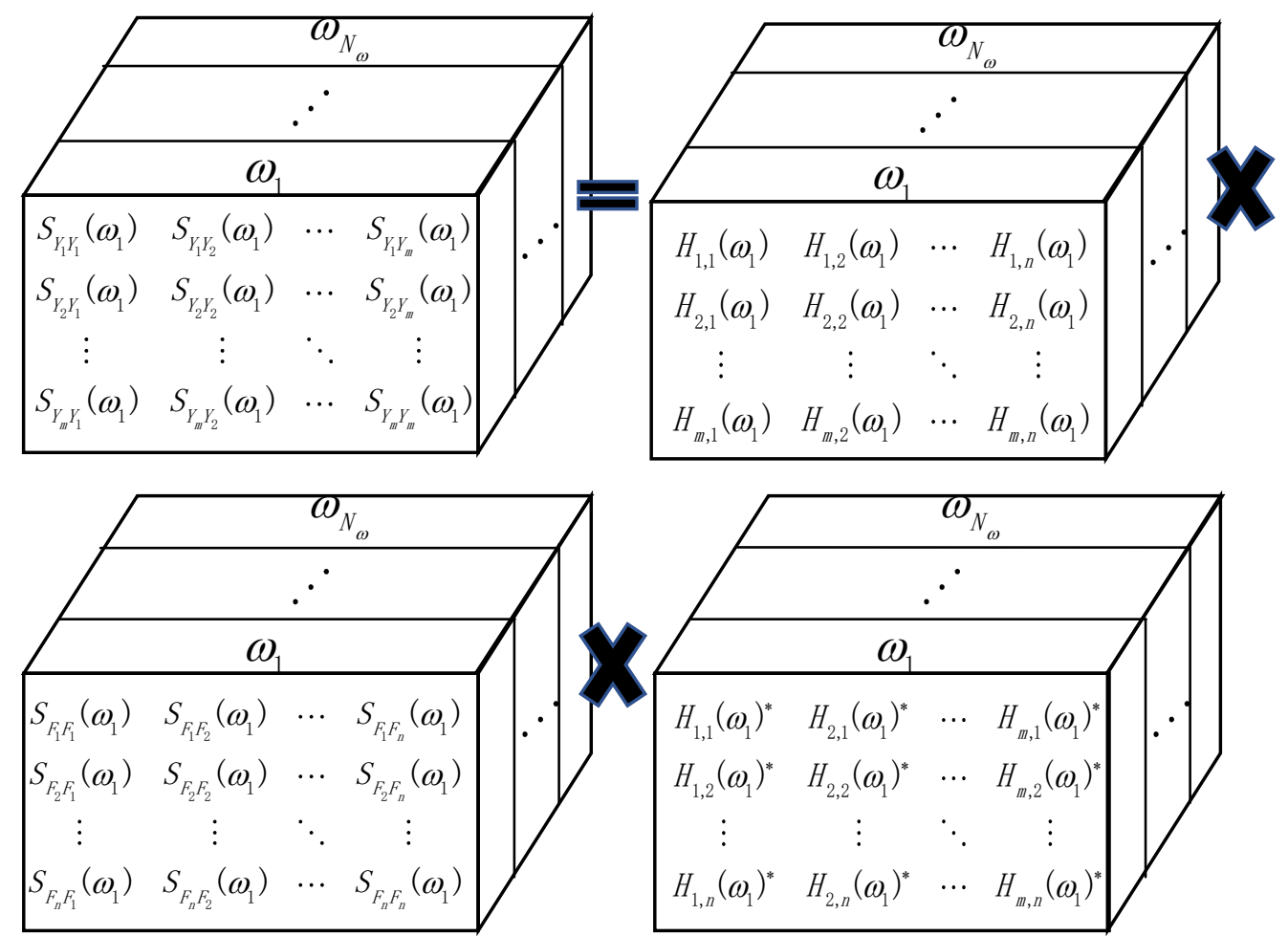

Fig. 1. The specific form of discrete random vibration formula. 
in which $\mathbf{S}_{\mathbf{F F}}(\omega) \in \mathbf{C}^{n \times n}$ and $\mathbf{S}_{\mathbf{Y Y}}(\omega) \in \mathbf{C}^{m \times m}$ are the PSD matrices of random excitation and dynamic responses, respectively. $\mathbf{H}(\omega) \in \mathbf{C}^{m \times n}$ denotes the FRF matrix. $\omega=\left(\omega_{1}, \omega_{2}, \cdots, \omega_{N_{\omega}}\right)$ represent the frequencies. Superscript $H$ is known as the complex conjugate transpose operator. Superscript $*$ stands for the complex conjugate operator.

Its equation of motion can be described as

$$
\mathbf{M} \ddot{\mathbf{y}}+\mathbf{C} \dot{\mathbf{y}}+\mathbf{K y}=\mathbf{F}(t)
$$

where $\mathbf{M}, \mathbf{C}$ and $\mathbf{K}$ are matrices of mass, damping and stiffness, respectively. $\mathbf{F}(t) \in \mathbf{C}^{n \times 1}$ is a stationary random dynamic load vector, in which PSD matrix $\mathbf{S}_{\mathbf{F F}}(\omega)$ is known. $t$ denotes the time. Since PSD matrix is a Hermit and non-negative definite matrix, it can be decomposed as follows:

$$
\mathbf{S}_{\mathbf{F F}}(\omega)=\sum_{j=1}^{r} \gamma_{j} \boldsymbol{\varphi}_{j} \boldsymbol{\varphi}_{j}{ }^{H}=\sum_{j=1}^{r} \tilde{\mathbf{F}}_{j}(\omega) \tilde{\mathbf{F}}_{j}(\omega)^{H} \quad(r \leq n)
$$

where $\gamma_{j}$ and $\boldsymbol{\varphi}_{j}$ are eigenvalue and corresponding eigenvector of PSD matrix $\mathbf{S}_{\mathbf{F F}}(\omega)$, $r$ is the rank of $\mathbf{S}_{\mathbf{F F}}(\omega), \tilde{\mathbf{F}}_{j}(\omega)=\sqrt{\gamma_{j}} \boldsymbol{\varphi}_{j}$, the superscript $\sim$ denotes the pseudo variables.

Thus, constituting $r$ independent pseudo harmonic excitations

$$
\tilde{\mathbf{f}}_{j}(t)=\tilde{\mathbf{F}}_{j}(\omega) e^{i \omega t}=\sqrt{\gamma_{j}} \boldsymbol{\varphi}_{j} e^{i \omega t} \quad(j=1,2, \ldots, r)
$$

Substituting Eq. (4) into Eq. (2) can transform the original complex system into a simple harmonic equation of motion, and the pseudo harmonic responses can be derived by modal superposition method.

$$
\tilde{\mathbf{y}}_{j}(t)=\tilde{\mathbf{Y}}_{j}(\omega) e^{i \omega t} \quad(j=1,2, \ldots, r)
$$

where $\tilde{\mathbf{Y}}_{j}(\omega)=\mathbf{H}(\omega) \tilde{\mathbf{F}}_{j}(\omega)$. 
The PSD matrix of dynamic responses can be expressed

$$
\mathbf{S}_{\mathbf{Y Y}}(\omega)=\sum_{j=1}^{r} \tilde{\mathbf{Y}}_{j}(\omega) \tilde{\mathbf{Y}}(\omega)_{j}{ }^{H}
$$

Eq. (1) can be reformulated as follows:

$$
\mathbf{S}_{\mathbf{F F}}(\omega)=\mathbf{H}(\omega)^{+} \mathbf{S}_{\mathbf{Y Y}}(\omega) \mathbf{H}(\omega)^{+H}
$$

where the superscript + denotes the generalized inversion. Obviously, Eq. (7) is the backward process of the pseudo excitation method (PEM) presented above. Consequently, the IPEM can be described as follows:

Decomposing $\mathbf{S}_{\mathbf{Y Y}}$ into $\sum_{j=1}^{r} \tilde{\mathbf{Y}}_{j}(\omega) \tilde{\mathbf{Y}}_{j}(\omega)^{H}$, the pseudo excitation can be obtained

$$
\begin{gathered}
\tilde{\mathbf{Y}}_{j}(\omega)=\mathbf{H}(\omega) \tilde{\mathbf{F}}_{j}(\omega) \quad(j=1,2, \ldots, r) \\
\tilde{\mathbf{F}}_{j}(\omega)=\mathbf{H}(\omega)^{+} \tilde{\mathbf{Y}}_{j}(\omega) \quad(j=1,2, \ldots, r)
\end{gathered}
$$

And then the PSD matrix of random excitation can be obtained as follows:

$$
\mathbf{S}_{\mathbf{F F}}(\omega)=\sum_{j=1}^{r} \tilde{\mathbf{F}}_{j}(\omega) \tilde{\mathbf{F}}_{j}(\omega)^{H}
$$

\subsection{Formulation of forward problem}

If some structural properties such as Young's modulus and Poisson's ratio are not completely determined, the FRF, which represents the dynamic behavior of structures, would have the stochastic characteristic. Under this circumstance, the PSD of random excitation to be identified is not a definite result but related to the random structural parameters. Hence, the FRF and identified random excitation are described as the functions of frequency and random structural parameters. The forward problem of combined random 
excitation identification can be written as

$$
\mathbf{S}_{\mathbf{Y Y}}(\omega)=\mathbf{H}(\omega, \boldsymbol{\eta}) \mathbf{S}_{\mathbf{F F}}(\omega, \boldsymbol{\eta}) \mathbf{H}(\omega, \boldsymbol{\eta})
$$

in which $\boldsymbol{\eta}$ denotes the $q$-dimensional random structural parameters vector.

Accordingly, the relation between pseudo excitations and pseudo responses can be rewritten as

$$
\tilde{\mathbf{Y}}_{j}(\omega)=\mathbf{H}(\omega, \boldsymbol{\eta}) \tilde{\mathbf{F}}_{j}(\omega, \boldsymbol{\eta})
$$

The PSD of random excitations can be obtained as follows:

$$
\mathbf{S}_{\mathbf{F F}}(\omega, \boldsymbol{\eta})=\sum_{j=1}^{r} \tilde{\mathbf{F}}_{j}(\omega, \boldsymbol{\eta}) \tilde{\mathbf{F}}_{j}(\omega, \boldsymbol{\eta})^{H}
$$

Consequently, the random dynamic loads for stochastic structures can be identified provided that the pseudo excitations are obtained by means of inversion of Eq. (11).

\section{Modified regularization method for random excitation identification with deterministic structural parameters}

\subsection{Error analysis and method to select good measurement locations}

In most practical engineering circumstances, the measurement noise is extensively contained in measuring dynamic responses. It has a significant effect on the results of random dynamic loads to be identified, which makes the inverse problem ill-posed. Therefore, the measuring dynamic responses can be reformulated as

$$
\mathbf{S}_{\mathbf{Y Y}}^{\boldsymbol{\delta}}(\omega)=\mathbf{S}_{\mathbf{Y Y}}(\omega)+\Delta \mathbf{S}_{\mathbf{Y Y}}(\omega)=\mathbf{H}(\omega) \mathbf{S}_{\mathbf{F F}}(\omega) \mathbf{H}(\omega)^{H}+\Delta \mathbf{S}_{\mathbf{Y Y}}(\omega)
$$

in which $\mathbf{S}_{\mathbf{Y Y}}^{\delta}$ is the PSD matrix of the measuring responses, $\mathbf{S}_{\mathbf{Y Y}}$ and $\Delta \mathbf{S}_{\mathbf{Y Y}}$ are the PSD matrices of actual dynamic responses and measurement noise respectively.

Accordingly, the equation for identifying random excitation can be rewritten as 


$$
\mathbf{S}_{\mathbf{Y Y}}^{\boldsymbol{\delta}}(\omega)=\mathbf{H}(\omega) \mathbf{S}_{\mathbf{F F}}^{\boldsymbol{\delta}}(\omega) \mathbf{H}(\omega)^{H}
$$

where $\mathbf{S}_{\mathbf{F F}}^{\boldsymbol{\delta}}(\omega)=\mathbf{S}_{\mathbf{F F}}(\omega)+\Delta \mathbf{S}_{\mathbf{F F}}(\omega), \mathbf{S}_{\mathbf{F F}}(\omega)$ and $\Delta \mathbf{S}_{\mathbf{F F}}(\omega)$ are the PSD matrices of actual random dynamic loads and error terms.

In consideration of the ill-posed problem of the inverse process caused by measurement noise and ill-conditioned FRF matrix, it is imperative to analyze the error source and the factors affecting the relative error of identified random loads. Otherwise, a large margin of error contained in the results of random excitation to be identified will greatly reduce its effectiveness and feasibility.

Comparing Eq. (13) with Eq. (14), the PSD matrix of identified random excitation can be written as

$$
\mathbf{S}_{\mathbf{F F}}^{\boldsymbol{\delta}}(\omega)=\mathbf{H}(\omega)^{+} \mathbf{S}_{\mathbf{Y Y}}^{\boldsymbol{\delta}}(\omega) \mathbf{H}(\omega)^{+H}=\mathbf{H}(\omega)^{+} \mathbf{S}_{\mathbf{Y Y}}(\omega) \mathbf{H}(\omega)^{+H}+\mathbf{H}(\omega)^{+} \Delta \mathbf{S}_{\mathbf{F F}}(\omega) \mathbf{H}(\omega)^{+H}
$$

Therefore, the error terms of identified random loads can be expressed as

$$
\Delta \mathbf{S}_{\mathbf{F F}}(\omega)=\mathbf{H}(\omega)^{+} \Delta \mathbf{S}_{\mathbf{F F}} \mathbf{H}(\omega)^{+H}
$$

In order to obtain the relative error of identified random excitation, the matrix norm is applied to Eq. (1) and Eq. (16):

$$
\begin{gathered}
\left\|\mathbf{S}_{\mathbf{Y Y}}(\omega)\right\| \leq\|\mathbf{H}(\omega)\| \mathbf{S}_{\mathbf{F F}}(\omega)\left\|\mathbf{H}(\omega)^{H}\right\| \\
\left\|\Delta \mathbf{S}_{\mathbf{F F}}(\omega)\right\| \leq\left\|\mathbf{H}(\omega)^{+}\right\|\left\|\Delta \mathbf{S}_{\mathbf{Y Y}}(\omega)\right\|\left\|\mathbf{H}(\omega)^{+H}\right\|
\end{gathered}
$$

in which $\|\cdot\|$ denotes the norm of matrix or vector.

Multiplying Eq. (17) by Eq. (18):

$\left\|\mathbf{S}_{\mathbf{Y Y}}(\omega)\right\|\left\|\Delta \mathbf{S}_{\mathbf{F F}}(\omega)\right\| \leq\left\|\mathbf{H}(\omega)^{+}\right\|\|\mathbf{H}(\omega)\|\left\|\Delta \mathbf{S}_{\mathbf{Y Y}}(\omega)\right\|\left\|\mathbf{S}_{\mathbf{F F}}(\omega) \mid\right\| \mathbf{H}(\omega)^{H}\left\|\mathbf{H}(\omega)^{+H}\right\|$

As a result, the relative error of identified random excitations can be derived 


$$
\frac{\left\|\Delta \mathbf{S}_{\mathbf{F F}}(\omega)\right\|}{\left\|\mathbf{S}_{\mathbf{F F}}(\omega)\right\|} \leq\left\|\mathbf{H}(\omega)^{+}\right\|\|\mathbf{H}(\omega)\|\left\|\mathbf{H}(\omega)^{H}\right\| \mathbf{H}(\omega)^{+H} \| \frac{\left\|\Delta \mathbf{S}_{\mathbf{Y Y}}(\omega)\right\|}{\left\|\mathbf{S}_{\mathbf{Y Y}}(\omega)\right\|}
$$

Based on the mathematical definition of condition number, Eq. (20) can be rewritten as

$$
\frac{\left\|\Delta \mathbf{S}_{\mathbf{F F}}(\omega)\right\|}{\left\|\mathbf{S}_{\mathbf{F F}}(\omega)\right\|} \leq \operatorname{cond}(\mathbf{H}(\omega)) \operatorname{cond}\left(\mathbf{H}(\omega)^{H}\right) \frac{\left\|\Delta \mathbf{S}_{\mathbf{Y Y}}(\omega)\right\|}{\left\|\mathbf{S}_{\mathbf{Y Y}}(\omega)\right\|}
$$

in which $\operatorname{cond}(\mathbf{H}(\omega))=\left\|\mathbf{H}(\omega)^{+}\right\| \mathbf{H}(\omega) \|, \quad \operatorname{cond}\left(\mathbf{H}(\omega)^{H}\right)=\operatorname{cond}(\mathbf{H}(\omega))$.

From Eq. (21), it can be inferred that the condition number cond $(\mathbf{H}(\omega))$ of FRF matrix and measuring noise $\frac{\left\|\Delta \mathbf{S}_{\mathbf{Y Y}}(\omega)\right\|}{\left\|\mathbf{S}_{\mathbf{Y Y}}(\omega)\right\|}$ are the key factors to affect the relative error of identified random excitation $\frac{\left\|\Delta \mathbf{S}_{\mathbf{F F}}(\omega)\right\|}{\left\|\mathbf{S}_{\mathbf{F F}}(\omega)\right\|}$. Hence, decreasing the condition number of FRF matrix is an effective and feasible means to mitigate the relative error of identified random excitation, which makes the ill-posed problem well-conditioned to a certain extent.

Due to the modal characteristic of structures, the condition number of FRF matrix mainly depends on the relative location of measuring responses points. Thus, it can prevent the condition number of FRF matrix from amplification by means of selecting proper response measurement locations. When there are plenty of optimal locations, for instance, selecting four positions from twenty available ones would have $C_{20}^{4}=4845$ combinations; moreover, the number of combinations would rise to $\mathrm{C}_{20}^{6}=38760$ for selecting six locations from twenty. Therefore, a huge amount of computation is involved in the event of determining the condition number for each of combination at each frequency. In order to solve this problem, a method based on composite condition number is employed and the procedure is described in detail as follows [38]: 
(1) Select $M$ locations as the candidates for the measurement responses.

(2) Pick two responses locations $(i, j)$ from $M$ ones and constitute a $2 \times N$ FRF matrix ( $N$ is the number of excitation positions to be identified), and calculate the condition numbers of the matrix at each frequency.

(3) Calculate the average value of these condition numbers over the considered frequency range, written as $a_{i j}$.

(4) Construct a $M \times M$ matrix based on the $a_{i j}$ ( $a_{i j}$ is the $j$ column of $i$ row in this matrix), and the diagonal terms of the matrix are zero.

(5) Determine $m$ responses for usage, and the number of combinations of $m$ taken from $M$ is $\mathrm{C}_{M}^{m}=\frac{M !}{m !(M-m) !}$.

(6) Construct a formula of composite condition numbers as: $A=\frac{2 !(m-2) !}{m !} \sum_{i, j} a_{i j}$.

(7) Calculate the composite condition numbers for all combinations determined in (5).

(8) The combination with minimum composite numbers is the optimum combination of measurement locations.

\subsection{Modified regularization method}

Considering the load identification, the ill-posed problems primarily result from the cluster of small singular values of FRF matrix. When the singular value tends to zero, the measuring noise in responses would be seriously amplified thereby leading to the instability and inaccuracy of identified loads. Therefore, in order to obtain a stable and useful solution, it is necessary to incorporate supplementary information about the identified loads, which is the aim of regularization. 


\subsubsection{Traditional regularization method}

On the basis of IPEM, the regularization method can be applied to the identification of pseudo excitation. And Eq. (8) can be transformed into a linear least-squares problem described in Eq. (22).

$$
\min _{\widetilde{\mathbf{F}}_{j}}\left\|\mathbf{H}(\omega) \tilde{\mathbf{F}}_{j}(\omega)-\tilde{\mathbf{Y}}_{j}(\omega)\right\|
$$

There is no doubt that the Tikhonov regularization is regarded as the most well-known and common form of regularization [39, 40]. This method seeks to determine a helpful approximation through replacing the minimization problem of Eq. (22) by a penalized least-squares problem of Eq. (23) as follows:

$$
\tilde{\mathbf{F}}_{j}^{\lambda}(\omega)=\arg \min \left\{\left\|\mathbf{H}(\omega) \tilde{\mathbf{F}}_{j}(\omega)-\tilde{\mathbf{Y}}_{j}(\omega)\right\|^{2}+\lambda^{2}\left\|\tilde{\mathbf{F}}_{j}(\omega)\right\|^{2}\right\}
$$

in which $\lambda$ denotes the regularization parameter, and the regular solution $\tilde{\mathbf{F}}_{j}^{\lambda}(\omega)$ defined by Eq. (23), can be solved by the least squares problem as follows:

$$
\min _{\tilde{\mathbf{F}}_{j}(\omega)}\left\|\left(\begin{array}{c}
\mathbf{H}(\omega) \\
\lambda \mathbf{I}
\end{array}\right) \tilde{\mathbf{F}}_{j}(\omega)-\left(\begin{array}{c}
\tilde{\mathbf{Y}}_{j}(\omega) \\
\mathbf{0}
\end{array}\right)\right\|
$$

Based on the theory of SVD [41], the FRF matrix can be decomposed as

$$
\mathbf{H}(\omega)=\mathbf{U} \boldsymbol{\Sigma} V^{H}=\sum_{i=1}^{n} \mathbf{u}_{i} \alpha_{i} \mathbf{v}_{i}^{H}
$$

in which $\mathbf{U}=\left(\mathbf{u}_{1}, \mathbf{u}_{2}, \cdots, \mathbf{u}_{m}\right), \mathbf{V}=\left(\mathbf{v}_{1}, \mathbf{v}_{2}, \cdots, \mathbf{v}_{n}\right), \mathbf{u}_{i}$ and $\mathbf{v}_{i}$ denote the left singular vectors and right singular vectors of FRF matrix $\mathbf{H}(\omega)$ respectively; and $\boldsymbol{\Sigma}=\operatorname{diag}\left(\alpha_{1}, \alpha_{2}, \cdots \alpha_{n}\right), \quad \alpha_{i}$ denotes the singular value of $\mathbf{H}(\omega)$ and has non-negative terms appearing in non-increasing order as $\alpha_{1} \geq \alpha_{2} \geq \cdots \geq \alpha_{n}$.

Eq. (24) can be rewritten as 


$$
\left(\mathbf{H}(\omega)^{H} \mathbf{H}(\omega)+\lambda^{2} \mathbf{I}\right) \tilde{\mathbf{F}}_{j}(\omega)=\mathbf{H}(\omega)^{H} \tilde{\mathbf{Y}}_{j}(\omega)
$$

From Eq. (26), the identified pseudo excitation can be obtained

$$
\tilde{\mathbf{F}}_{j}(\omega)=\left(\mathbf{H}(\omega)^{H} \mathbf{H}(\omega)+\lambda^{2} \mathbf{I}\right)^{-1} \mathbf{H}(\omega)^{H} \tilde{\mathbf{Y}}_{j}(\omega)
$$

Clearly, owing to putting the non-negative elements $\lambda^{2} \mathbf{I}$ in diagonal of $\mathbf{H}(\omega)^{H} \mathbf{H}(\omega)$, it tremendously simplifies the difficulties of inversion for $\left(\mathbf{H}(\omega)^{H} \mathbf{H}(\omega)+\lambda^{2} \mathbf{I}\right)$ compared with $\mathbf{H}(\omega)^{H} \mathbf{H}(\omega)$. Comparing the condition number of $\left(\mathbf{H}(\omega)^{H} \mathbf{H}(\omega)+\lambda^{2} \mathbf{I}\right)$ with $\mathbf{H}(\omega)^{H} \mathbf{H}(\omega)$

$$
\operatorname{cond}\left(\mathbf{H}(\omega)^{H} \mathbf{H}(\omega)+\lambda^{2} \mathbf{I}\right)=\frac{\alpha_{\max }^{2}+\lambda^{2}}{\alpha_{\min }^{2}+\lambda^{2}}<\frac{\alpha_{\max }^{2}}{\alpha_{\min }^{2}}=\operatorname{cond}\left(\mathbf{H}(\omega)^{H} \mathbf{H}(\omega)\right)
$$

Consequently, when the FRF matrix is ill-conditioned, it can immensely overcome the ill-posed problems of load identification by means of replacing the original ill-conditioned operator $\mathbf{H}(\omega)^{H} \mathbf{H}(\omega)$ by the approximate operator $\left(\mathbf{H}(\omega)^{H} \mathbf{H}(\omega)+\lambda^{2} \mathbf{I}\right)$.

Based on Eq. (25), Eq. (27) can also be reformulated as

$$
\tilde{\mathbf{F}}_{j}(\omega)=\mathbf{V} \operatorname{diag}\left(f\left(\lambda, \alpha_{i}\right) \alpha_{i}^{-1}\right) \mathbf{U}^{H} \tilde{\mathbf{Y}}_{j}(\omega)=\sum_{i=1}^{n} f\left(\lambda, \alpha_{i}\right) \alpha_{i}^{-1}\left(\mathbf{u}_{i}^{H} \tilde{\mathbf{Y}}_{j}(\omega)\right) \mathbf{v}_{i}
$$

where $f\left(\lambda, \alpha_{i}\right)$ denotes the regularization operator, as regards Tikhonov regularization, it can be described as

$$
f\left(\lambda, \alpha_{i}\right)=\frac{\alpha_{i}^{2}}{\lambda^{2}+\alpha_{i}^{2}}
$$

Assuming that the regularization operator is taken as

$$
f\left(\lambda, \alpha_{i}\right)= \begin{cases}1, & \alpha_{i} \geq \lambda \\ 0, & \alpha_{i}<\lambda\end{cases}
$$

Thus, the truncated singular value decomposition (TSVD) can be derived, and the corresponding regularization solution can be expressed as 


$$
\tilde{\mathbf{F}}_{j}(\omega)=\sum_{\alpha_{i} \geq \lambda} \alpha_{i}^{-1}\left(\mathbf{u}_{i}^{H} \tilde{\mathbf{Y}}_{j}(\omega)\right) \mathbf{v}_{i}
$$

\subsubsection{A novel modified regularization parameter}

According to the theorem of the regularization [42], it is known that the regularization parameter $\lambda$ introduced by Eq. (23), controls the weight applied to minimization of the residual norm with respect to minimization of side constraint. From Eq. (29), we can infer that

(1) $\tilde{\mathbf{F}}_{j}(\omega)$ depends continuously on $\tilde{\mathbf{Y}}_{j}(\omega)$ and $\lambda$;

(2) The solution norm $\left\|\widetilde{\mathbf{F}}_{j}(\omega)\right\|$ is monotone non-increasing with $\lambda$, $\lim _{\lambda \rightarrow \infty}\left\|\widetilde{\mathbf{F}}_{j}(\omega)\right\|=0 ;$

(3) The residual norm $\left\|\mathbf{H}(\omega) \tilde{\mathbf{F}}_{j}(\omega)-\tilde{\mathbf{Y}}_{j}(\omega)\right\|$ is monotone non-decreasing with $\lambda$.

Obviously, a small $\lambda$ (namely a small quantity of regularization) leads to a small residual norm at the expense of a large solution norm, thus ensuring the approximation between the measuring responses and the evaluating ones, whereas a large $\lambda$ has the effect oppositely so as to ensure the stability of identified solutions. It also can be inferred that the sensitivity of the regular solutions of identified load to perturbations in the FRF matrix $\mathbf{H}(\omega)$ and response vector are regulated by $\lambda$. Consequently, it is a matter of cardinal significance that singling out the appropriate $\lambda$ to equilibrate the precision and stability of the approximate solution, which is also the essential issue of regularization methods. In what follows, the numerical methods for choosing the regularization parameter $\lambda$ are introduced, and a novel modified regularization parameter based on IPEM is proposed for random dynamic loads identification. 
At present, numerous criterions such as Morozov deviation principle, Engl criterion, quasi-optimal criterion, generalized cross-validation (GCV) criterion and L-curve criterion have been put forward to determine the optimal regularization parameters, among which GCV criterion is the most widely used method.

The GCV criterion can be expressed as follows:

$$
\mathrm{V}(\lambda)=\frac{\left\|\mathbf{H}(\omega) \tilde{\mathbf{F}}_{j}(\omega)-\tilde{\mathbf{Y}}_{j}(\omega)\right\|^{2}}{\operatorname{tr}\left[\mathbf{I}-\mathbf{H}(\omega)\left(\mathbf{H}(\omega)^{H} \mathbf{H}(\omega)+\lambda^{2} \mathbf{I}\right)^{-1} \mathbf{H}(\omega)^{H}\right)^{2}}
$$

in which $\operatorname{tr}[\cdot]$ denotes the matrix trace, namely the sum of matrix diagonal elements. The regularization parameter can be obtained by determining the minimum point of $V(\lambda)$.

In the light of the characteristic of pseudo excitation and effects of the regularization parameter $\lambda$ on the residual norm and solution norm, each order of pseudo responses vector contains the energy or the information of the measuring responses PSD matrix at different level. The energy of the pseudo response vector is strongly related to the eigenvalue $\sigma_{j}$ of the response PSD matrix and monotonically decreasing with orders. Thus, the first order of pseudo response contains most of the information, so the regularization parameter should be small in order to preserve more information of measuring responses and ensure the accuracy of the identified random excitation. As the posterior orders of pseudo responses contain less information and the measuring noise has a great influence on stability of identified solutions, the regularization parameter should be large so as to ensure the stability of identified random excitation. Consequently, a novel modified regularization parameter is proposed as follows: 


$$
\lambda_{j}^{\mathrm{M}}=\frac{\operatorname{cond}(\mathbf{H}(\omega)) \sqrt{\sigma_{1}} \lambda}{\operatorname{cond}(\mathbf{H}(\omega))_{\max } \sqrt{\sigma_{j}}}
$$

where $\lambda_{j}^{\mathrm{M}}$ denotes the modified regularization parameter for $j$ order of pseudo response, $\sigma_{j}$ is the eigenvalue of measuring responses PSD matrix, $\lambda$ is obtained by means of GCV, and $\operatorname{cond}(\mathbf{H}(\omega))_{\max }$ is the maximum value of condition number of FRF matrix over the frequency range.

It is noted from Eq. (34) that the proposed modified regularization parameter is strictly controlled by the condition number of FRF matrix as well as the eigenvalue of measuring responses PSD matrix. Firstly, since different orders of pseudo responses vector contain different amounts of information of the measuring responses PSD matrix, which is the major difference between random load identification and deterministic load identification, the $\frac{\sqrt{\sigma_{1}}}{\sqrt{\sigma_{j}}}$ is employed to adjust the $\lambda_{j}^{\mathrm{M}}$ to match the pseudo responses well. On that basis, the pseudo responses containing a large amount of information match a small regularization parameter resulting in preserving more information of measuring responses and ensuring the precision of identified random excitation. On the contrary, the pseudo responses containing less information match a large regularization parameter leading to reduce the effect of the measuring noise on stability of the identified solutions. Additionally, based on the mathematical deviation of the relative error formula of identified random loads in Section 3.1, it is seen that the relative error of identified random excitation is strongly associated with the condition number of FRF matrix. Thus, the $\frac{\operatorname{cond}(\mathbf{H}(\omega))}{\operatorname{cond}(\mathbf{H}(\omega))_{\max }}$ is introduced to adjust the $\lambda_{j}^{\mathrm{M}}$ to solve the inversion problems with different condition 
number of FRF matrix over the whole frequency range. Based on that, the large condition number of FRF matrix in the vicinity of natural frequencies, especially in the low frequency region $[35,43]$, construct a large regularization parameter to ensure the stability of identified random excitation in the low frequency range. The relatively small condition number of FRF matrix in the other frequency range constructs a small regularization parameter so as to prevent the identified results from over regularization.

Substituting the modified regularization parameter into Eq. (29), the identified pseudo excitation can be obtained

$$
\tilde{\mathbf{F}}_{j}^{\mathrm{M}}(\omega)=\mathbf{V} \operatorname{diag}\left(f\left(\lambda_{j}^{\mathrm{M}}, \alpha_{i}\right) \alpha_{i}^{-1}\right) \mathbf{U}^{H} \tilde{\mathbf{Y}}_{j}(\omega)=\sum_{i=1}^{n} f\left(\lambda_{j}^{\mathrm{M}}, \alpha_{i}\right) \alpha_{i}^{-1}\left(\mathbf{u}_{i}^{H} \tilde{\mathbf{Y}}_{j}(\omega)\right) \mathbf{v}_{i}
$$

In this paper, the modified regularization method is based on Tikhonov regularization operator, namely $f\left(\lambda_{j}^{\mathrm{M}}, \alpha_{i}\right)=\frac{\alpha_{i}^{2}}{\lambda_{j}^{\mathrm{M}^{2}}+\alpha_{i}^{2}}$.

Accordingly, the PSD matrix of the identified random excitation can be written as follows:

$$
\mathbf{S}_{\mathbf{F F}}(\omega)=\sum_{j=1}^{r} \tilde{\mathbf{F}}_{j}^{\mathrm{M}}(\omega) \tilde{\mathbf{F}}_{j}^{\mathrm{M}}(\omega)^{H}
$$

\section{Matrix perturbation for combined random excitation identification}

Considering the randomness of the FRF, Eq. (11) can be solved through MCS method, which is able to give accurate solutions but requires a huge computational cost leading to a low computational efficiency. Therefore, the matrix perturbation method is applied to transform the combined random excitation identification into a series of deterministic problems. 


\subsection{Sensitivities of pseudo excitation with respect to uncertain structural parameters}

Assuming that the random structural parameters fluctuate slightly, the vector of random structural parameter $\boldsymbol{\eta}=\left(\eta_{1}, \eta_{2}, \cdots, \eta_{q}\right)$ can be expressed as follows:

$$
\boldsymbol{\eta}=\boldsymbol{\eta}_{m}+\Delta \boldsymbol{\eta}_{p}, \quad \eta_{l}=\eta_{m l}+\Delta \eta_{p l} \quad(l=1,2,3, \ldots, q)
$$

where the subscripts $m$ and $p$ denote the mean value and perturbation of random parameters, respectively. In the light of perturbation theory, the pseudo excitation to be identified and FRF matrix can be reformulated as follows:

$$
\begin{aligned}
\tilde{\mathbf{F}}_{j}(\omega, \boldsymbol{\eta}) & =\tilde{\mathbf{F}}_{j m}(\omega)+\Delta \tilde{\mathbf{F}}_{j p}(\omega) \\
\mathbf{H}(\omega, \boldsymbol{\eta}) & =\mathbf{H}_{m}(\omega)+\Delta \mathbf{H}_{p}(\omega)
\end{aligned}
$$

Substituting Eq. (38) and Eq. (39) into Eq. (11), it becomes

$$
\tilde{\mathbf{Y}}_{j}(\omega)=\left(\mathbf{H}_{m}(\omega)+\Delta \mathbf{H}_{p}(\omega)\right)\left(\tilde{\mathbf{F}}_{j m}(\omega)+\Delta \tilde{\mathbf{F}}_{j p}(\omega)\right)
$$

Expanding Eq. (40) and comparing the terms, we have

$$
\begin{gathered}
\tilde{\mathbf{Y}}_{j}(\omega)=\mathbf{H}_{m}(\omega) \tilde{\mathbf{F}}_{j m}(\omega) \\
-\Delta \mathbf{H}_{p}(\omega) \tilde{\mathbf{F}}_{j m}(\omega)=\mathbf{H}_{m}(\omega) \Delta \tilde{\mathbf{F}}_{j p}(\omega)
\end{gathered}
$$

where $\mathbf{H}_{m}(\omega)$ and $\tilde{\mathbf{Y}}_{j}(\omega)$ obtained by decomposition of $\mathbf{S}_{\mathbf{Y Y}}(\omega)$, are the deterministic values. Therefore, $\tilde{\mathbf{F}}_{j m}(\omega)$ can be derived by means of the modified regularization method for the deterministic structure. And then substituting $\tilde{\mathbf{F}}_{j m}(\omega)$ into Eq. (42), $\Delta \widetilde{\mathbf{F}}_{j p}(\omega)$ can be obtained subsequently. Solving Eq. (42) directly is not an expedient way, so the method on the basis of Taylor series expansion is applied as follows:

Since $\Delta \eta_{p l}$ is much smaller than $\eta_{m l}, \tilde{\mathbf{F}}_{j}(\omega, \boldsymbol{\eta})$ and $\mathbf{H}(\omega, \boldsymbol{\eta})$ can be expanded near the mean value of random structural parameters by using the first order approximation of Taylor formula, it becomes 


$$
\begin{array}{rr}
\Delta \widetilde{\mathbf{F}}_{j p}(\omega) \approx \sum_{l=1}^{q} \frac{\partial \tilde{\mathbf{F}}_{j}\left(\omega, \eta_{l}\right)}{\partial \eta_{l}} \Delta \eta_{p l} & (l=1,2,3, \ldots, q) \\
\Delta \mathbf{H}_{p}(\omega) \approx \sum_{l=1}^{q} \frac{\partial \mathbf{H}\left(\omega, \eta_{l}\right)}{\partial \eta_{l}} \Delta \eta_{p l} & (l=1,2,3, \ldots, q)
\end{array}
$$

in which $\frac{\partial \tilde{\mathbf{F}}_{j}\left(\omega, \eta_{l}\right)}{\partial \eta_{l}}$ and $\frac{\partial \mathbf{H}\left(\omega, \eta_{l}\right)}{\partial \eta_{l}}$ denote the partial derivative of $\tilde{\mathbf{F}}_{j}\left(\omega, \eta_{l}\right)$ and $\mathbf{H}\left(\omega, \eta_{l}\right)$ with respect to random structural parameter $\eta_{l}$ at the mean value $\eta_{m l}$, respectively. Substituting Eq. (43) and Eq. (44) into Eq. (42), and then comparing the coefficients on both sides of the equation, we have

$$
-\frac{\partial \mathbf{H}\left(\omega, \eta_{l}\right)}{\partial \eta_{l}} \tilde{\mathbf{F}}_{j m}(\omega)=\mathbf{H}_{m}(\omega) \frac{\partial \tilde{\mathbf{F}}_{j}\left(\omega, \eta_{l}\right)}{\partial \eta_{l}} \quad(l=1,2,3, \ldots, q)
$$

As a result, based on the matrix perturbation method described above, the problem of pseudo excitation identification for stochastic structures with $q$ random parameters is transformed into $q+1$ deterministic problems as follows:

$$
\begin{gathered}
\tilde{\mathbf{Y}}_{j}(\omega)=\mathbf{H}_{m}(\omega) \tilde{\mathbf{F}}_{j m}(\omega) \\
-\frac{\partial \mathbf{H}\left(\omega, \eta_{l}\right)}{\partial \eta_{l}} \tilde{\mathbf{F}}_{j m}(\omega)=\mathbf{H}_{m}(\omega) \frac{\partial \tilde{\mathbf{F}}_{j}\left(\omega, \eta_{l}\right)}{\partial \eta_{l}} \quad(l=1,2,3, \ldots, q)
\end{gathered}
$$

The $q+1$ deterministic problems described in Eq. (46) and Eq. (47) are two types of deterministic inverse problems, namely the pseudo excitation identification on the mean value of structural random parameters and the sensitivity identification of pseudo excitation with respect to each random parameter.

\subsection{Sensitivities of random excitation with respect to uncertain structural parameters}

Since the pseudo excitation on the mean value of random structural parameters $\tilde{\mathbf{F}}_{j m}(\omega)$ is obtained by Eq. (46), consequently, the PSD matrix of random dynamic loads on 
the mean value $\overline{\mathbf{S}}_{F F}(\omega)$ can be acquired as follows:

$$
\overline{\mathbf{S}}_{\mathbf{F F}}(\omega)=\sum_{j=1}^{r} \tilde{\mathbf{F}}_{j m}(\omega) \tilde{\mathbf{F}}_{j m}(\omega)^{H}
$$

Based on the result of sensitivity of pseudo excitation with respect to each random parameter, taking partial derivative of both sides of Eq. (12) with respect to random structural parameter $\eta_{l}$, the sensitivities of random excitation with respect to uncertain structural parameters $\eta_{l}$ can be derived

$$
\begin{array}{r}
\frac{\partial \mathbf{S}_{\mathbf{F F}}\left(\omega, \eta_{l}\right)}{\partial \eta_{l}}=\sum_{j=1}^{r} \frac{\partial \tilde{\mathbf{F}}_{j}\left(\omega, \eta_{l}\right)}{\partial \eta_{l}}\left\{\tilde{\mathbf{F}}_{j}\left(\omega, \eta_{l}\right)\right\}^{H}+\sum_{j=1}^{r}\left\{\tilde{\mathbf{F}}_{j}\left(\omega, \eta_{l}\right)\right\} \frac{\partial\left\{\tilde{\mathbf{F}}_{j}\left(\omega, \eta_{l}\right)\right\}^{H}}{\partial \eta_{l}} \\
(l=1,2,3, \ldots, q)
\end{array}
$$

\subsection{Statistical characteristic of identified random excitation for stochastic structures}

Utilizing the perturbation analysis and modified regularization method described in previous section, the random dynamic loads on the mean value and sensitivities of random excitation with respect to uncertain structural parameters can be obtained steadily and approximately. In order to show the relationship between identified random excitation results and structural random parameters intuitively, the statistical characteristic and the bounds of identified excitation are presented in this section.

Based on perturbation theory and first order Taylor series expansion, the random excitation to be identified and its fluctuation can be described in the same form of Eq. (38) and Eq. (43) respectively.

$$
\begin{gathered}
\mathbf{S}_{\mathbf{F F}}(\omega, \boldsymbol{\eta})=\overline{\mathbf{S}}_{\mathbf{F F}}(\omega)+\Delta \mathbf{S}_{\mathbf{F F}_{p}}(\omega) \\
\Delta \mathbf{S}_{\mathbf{F F}_{p}}(\omega) \approx \sum_{l=1}^{q} \frac{\partial \mathbf{S}_{\mathbf{F F}}\left(\omega, \eta_{l}\right)}{\partial \eta_{l}} \Delta \eta_{p l} \quad(l=1,2,3, \ldots, q)
\end{gathered}
$$


The expectation and variance of identified random dynamic loads can be obtained as follows

$$
\begin{aligned}
& \mathrm{E}\left[\mathbf{S}_{\mathbf{F F}}(\omega, \boldsymbol{\eta})\right]=\mathrm{E}\left[\overline{\mathbf{S}}_{\mathbf{F F}}(\omega)\right]+\mathrm{E}\left[\Delta \mathbf{S}_{\mathbf{F F}_{p}}(\omega)\right]=\overline{\mathbf{S}}_{\mathbf{F F}}(\omega) \\
& \operatorname{var}\left(\mathbf{S}_{\mathbf{F F}}(\omega, \boldsymbol{\eta})\right)=\int_{-\infty}^{\infty} \ldots \int_{-\infty}^{\infty}\left(\mathbf{S}_{\mathbf{F F}}(\omega, \boldsymbol{\eta})-\mathrm{E}\left[\mathbf{S}_{\mathbf{F F}}(\omega, \boldsymbol{\eta})\right]\right)^{2} \mathrm{f}(\boldsymbol{\eta}) d \boldsymbol{\eta} \\
& \approx \int_{-\infty}^{\infty} \ldots \int_{-\infty}^{\infty}\left(\sum_{l=1}^{q} \frac{\partial \mathbf{S}_{\mathbf{F F}}\left(\omega, \eta_{l}\right)}{\partial \eta_{l}} \Delta \eta_{p l}\right)^{2} \mathrm{f}(\boldsymbol{\eta}) d \boldsymbol{\eta} \\
&=\sum_{l_{1}}^{q} \sum_{l_{2}}^{q} \frac{\partial \mathbf{S}_{\mathbf{F F}}\left(\omega, \eta_{l_{1}}\right)}{\partial \eta_{l_{1}}} \frac{\partial \mathbf{S}_{\mathbf{F F}}\left(\omega, \eta_{l_{2}}\right)}{\partial \eta_{l_{2}}} \operatorname{cov}\left(\eta_{l_{1}}, \eta_{l_{2}}\right)
\end{aligned}
$$

where $f(\boldsymbol{\eta})$ is the joint probability density function of structural random parameters, $\operatorname{cov}\left(\eta_{l_{1}}, \eta_{l_{2}}\right)$ is the covariance of structural random parameters $\eta_{l_{1}}$ and $\eta_{l_{2}}$, namely

$$
\begin{aligned}
\operatorname{cov}\left(\eta_{l_{1}}, \eta_{l_{2}}\right) & =\int_{-\infty}^{\infty} \ldots \int_{-\infty}^{\infty} \Delta \eta_{p l_{1}} \Delta \eta_{p l_{2}} \mathrm{f}(\boldsymbol{\eta}) d \boldsymbol{\eta} \\
& =\rho_{l_{1} l_{2}} \operatorname{std}\left(\eta_{l_{1}}\right) \operatorname{std}\left(\eta_{l_{2}}\right)
\end{aligned}
$$

in which $\rho_{l_{1} l_{2}}$ denotes the correlation coefficients of $\eta_{l_{1}}$ and $\eta_{l_{2}}, \operatorname{std}\left(\eta_{l_{j}}\right)$ is the standard deviation of $\eta_{l_{j}}$. As $\eta_{l_{1}}$ and $\eta_{l_{2}}$ are uncorrelated, the variance of identified random excitation can be expressed as

$$
\operatorname{var}\left(\mathbf{S}_{\mathbf{F F}}(\omega, \boldsymbol{\eta})\right)=\sum_{l=1}^{q}\left(\frac{\partial \mathbf{S}_{\mathbf{F F}}\left(\omega, \eta_{l}\right)}{\partial \eta_{l}} \operatorname{std}\left(\eta_{l}\right)\right)^{2}
$$

In order to observe the effect of the structural random parameters on the result of identified random excitation visually, the bounds of random dynamic loads to be identified are formulated as follows:

$$
\begin{array}{r}
\mathbf{S}_{\mathbf{F F}}^{\mathbf{U}}(\omega)=\mathrm{E}\left[\mathbf{S}_{\mathbf{F F}}(\omega, \boldsymbol{\eta})\right]+2 \sqrt{\operatorname{var}\left(\mathbf{S}_{\mathbf{F F}}(\omega, \boldsymbol{\eta})\right)} \\
\mathbf{S}_{\mathbf{F F}}^{\mathbf{L}}(\omega)=\mathrm{E}\left[\mathbf{S}_{\mathbf{F F}}(\omega, \boldsymbol{\eta})\right]-2 \sqrt{\operatorname{var}\left(\mathbf{S}_{\mathbf{F F}}(\omega, \boldsymbol{\eta})\right)}
\end{array}
$$

where $\mathbf{S}_{\mathbf{F F}}^{\mathbf{U}}(\omega)$ and $\mathbf{S}_{\mathbf{F F}}^{\mathbf{L}}(\omega)$ denote the upper bound and lower bound of identified random 
dynamic loads respectively.

\section{Numerical Examples}

To validate the effectiveness and feasibility of proposed methods in this section, a cantilever plate problem is firstly studied to clarify how to identify random excitation by modified regularization method and matrix perturbation method, and the identified results are compared with those by Moore-Penrose pseudo inverse method and Tikhonov regularization method. Then, the proposed methods are applied to a vehicle door. Finally, a vehicle trim body problem is investigated to validate the applicability and superiority of the proposed modified regularization method.

\subsection{Procedure of random excitation identification}

Based on matrix perturbation method and modified regularization method, the procedure of random excitation identification for stochastic structures can be described as the following steps:

(1) Select the best combination of measurement locations with minimum composite condition number of FRF matrix.

(2) Calculate the responses $\mathbf{S}_{\mathbf{Y Y}}(\omega)$ based on given random excitation and finite element analysis, and then add a noise to the computational responses PSD matrix, and decompose the responses PSD matrix $\mathbf{S}_{\mathbf{Y Y}}^{\boldsymbol{\delta}}(\omega)$ into pseudo response vector $\tilde{\mathbf{Y}}_{j}(\omega)$.

(3) Decompose the FRF matrix $\mathbf{H}(\omega)$ by means of SVD and calculate the modified regularization parameter $\lambda_{j}^{\mathrm{M}}$ from Eq. (34). 
(4) Calculate the pseudo excitation $\tilde{\mathbf{F}}_{j m}(\omega)$ and random excitation PSD $\overline{\mathbf{S}}_{\mathbf{F F}}(\omega)$ on mean value of stochastic parameters from Eq. (35) and Eq. (48) respectively.

(5) Compute the sensitivities of pseudo excitation and random excitation with respect to uncertain structural parameters from Eq. (47) and Eq. (49) respectively.

(6) Determine the upper bound $\mathbf{S}_{\mathbf{F F}}^{\mathbf{U}}(\omega)$ and lower bound $\mathbf{S}_{\mathbf{F F}}^{\mathbf{L}}(\omega)$ of random excitation from Eq. (56).

\subsection{A cantilever plate problem}

\subsubsection{Simulation-based validation for modified regularization method}

In order to verify whether the proposed modified regularization method is technically feasible and effective to identify the random dynamic load, a simulation-based validation is investigated on a cantilever plate. As shown in Fig. 2, the size of the plate, namely the length, width, and thickness are $768 \times 368 \times 8 \mathrm{~mm}$, respectively. The properties of the plate materials are set as follows: the Young's modulus $E=71 \mathrm{GPa}$, the Poisson's ratio $v=0.31$, the material density $\rho=2700 \mathrm{~kg} / \mathrm{m}^{3}$, and the structural damping loss factor $\mu=0.06$. The simulation plate is preprocessed with 4416 quadrilateral meshes, of which the size is $8 \times 8 \mathrm{~mm}$. One side of the plate is fully constrained, and the others are free. Based on the modal analysis theory and by using finite element software, the modal parameter can be obtained. The modal frequencies are listed in Table 1.

\section{Table 1}

The modal frequencies of the cantilever plate.

\begin{tabular}{lcccccc}
\hline Mode & 1 & 2 & 3 & 4 & 5 & 6 \\
\hline Frequency $(\mathrm{Hz})$ & 11.52 & 50.96 & 71.80 & 165.26 & 201.48 & 315.24 \\
\hline
\end{tabular}




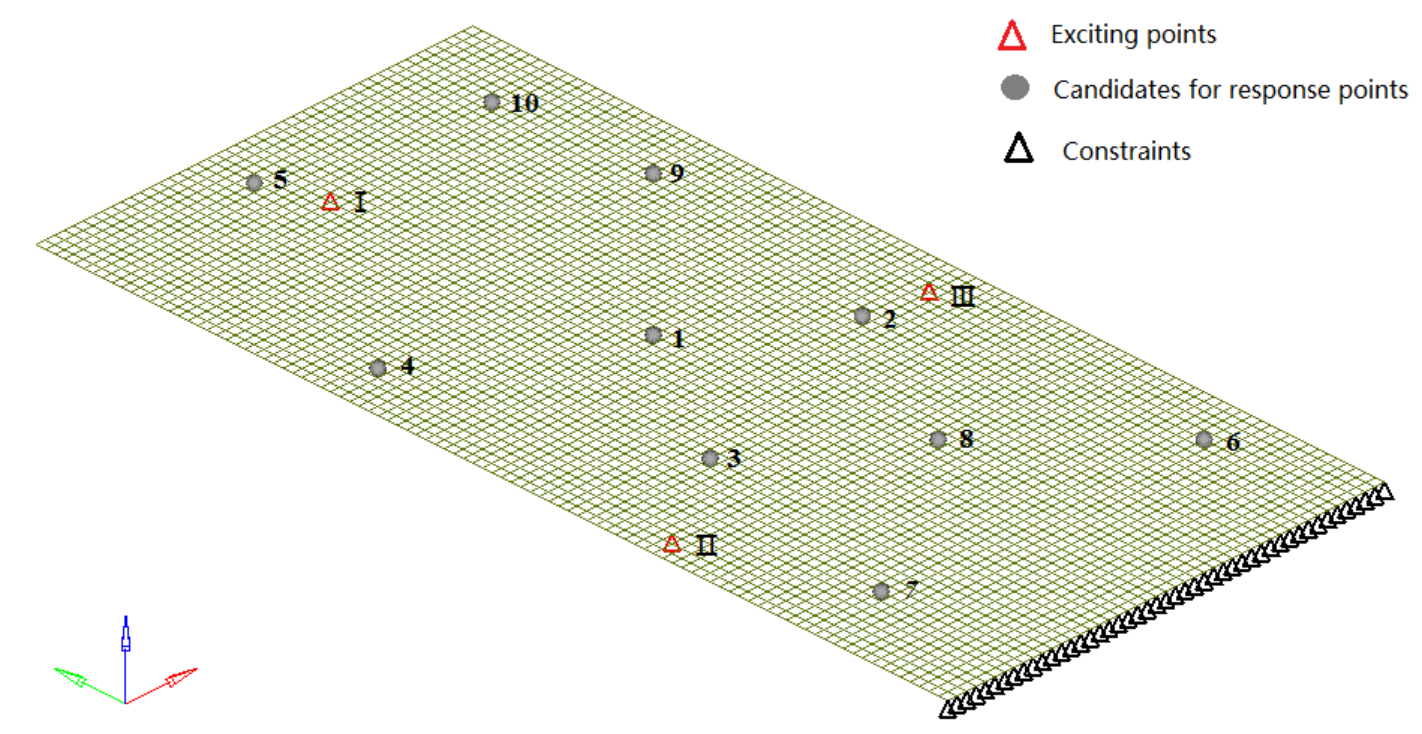

Fig. 2. The location of exciting points and candidates of response points.

Three exciting points and ten candidates for responses points are selected in $z$-direction at random. To mitigate the error propagation resulting from the large condition number of the FRF, the method of composite condition number is applied to select the best combination of five measurement locations from ten ones. Thus, by comparing the composite condition number of each optional location combination computed over the frequency region (from $0 \mathrm{~Hz}$ to $500 \mathrm{~Hz}$ ), the best and worst combinations of measurement locations are 1,3,5,8,9 and 2,6,8,9,10 respectively. As outlined in Fig. 3, it can be seen that the best combination is capable of reducing the condition number effectively compared with the worst combination. Especially close-by the modal frequencies and in the low frequency region, it decreases more markedly and tremendously. The peak values of condition numbers at the modal frequencies are listed in Table 2. We can infer that the error of identified random excitation would be amplified and propagated in the vicinity of natural frequencies owing to the large condition numbers of FRF matrix. 
Based on the given constructed PSD matrix of partial correlated stationary random excitation and finite element analysis, the random dynamic acceleration responses are computed. In order to simulate the actual measurement responses, a noise is added to the computational responses as shown in Fig. 4.

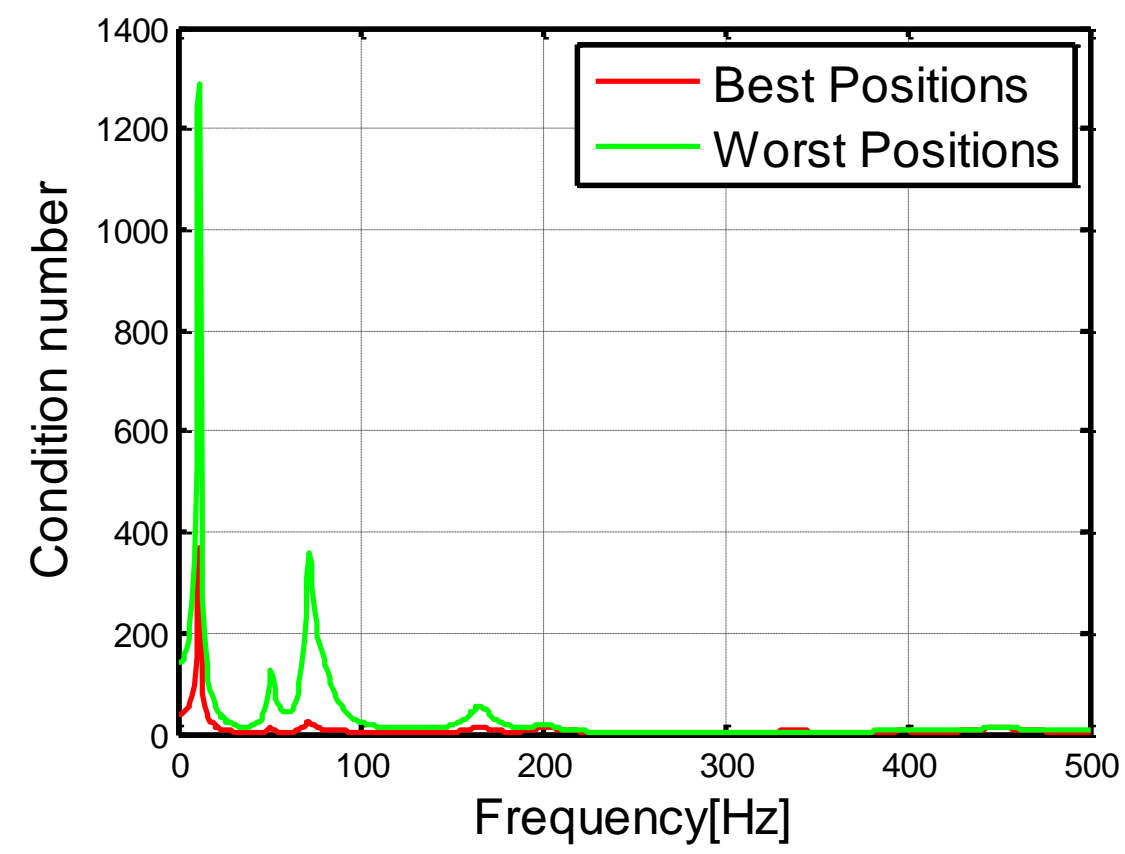

Fig. 3. Comparison of the FRF matrix condition number between best combination of measuring locations and worst one.

\section{Table 2}

The condition number of FRF matrix at the modal frequencies.

\begin{tabular}{ccccccc}
\hline Condition numbers & $\begin{array}{c}\text { Modal } \\
\text { freq 1 }\end{array}$ & $\begin{array}{c}\text { Modal } \\
\text { freq 2 }\end{array}$ & $\begin{array}{c}\text { Modal } \\
\text { freq 3 }\end{array}$ & $\begin{array}{c}\text { Modal } \\
\text { freq 4 }\end{array}$ & $\begin{array}{c}\text { Modal } \\
\text { freq 5 }\end{array}$ & $\begin{array}{c}\text { Modal } \\
\text { freq 6 }\end{array}$ \\
\hline Best combinations & 368.72 & 11.10 & 25.30 & 12.95 & 13.12 & 5.12 \\
Worst combinations & 1290.18 & 128.80 & 356.79 & 56.56 & 19.14 & 5.12 \\
\hline
\end{tabular}




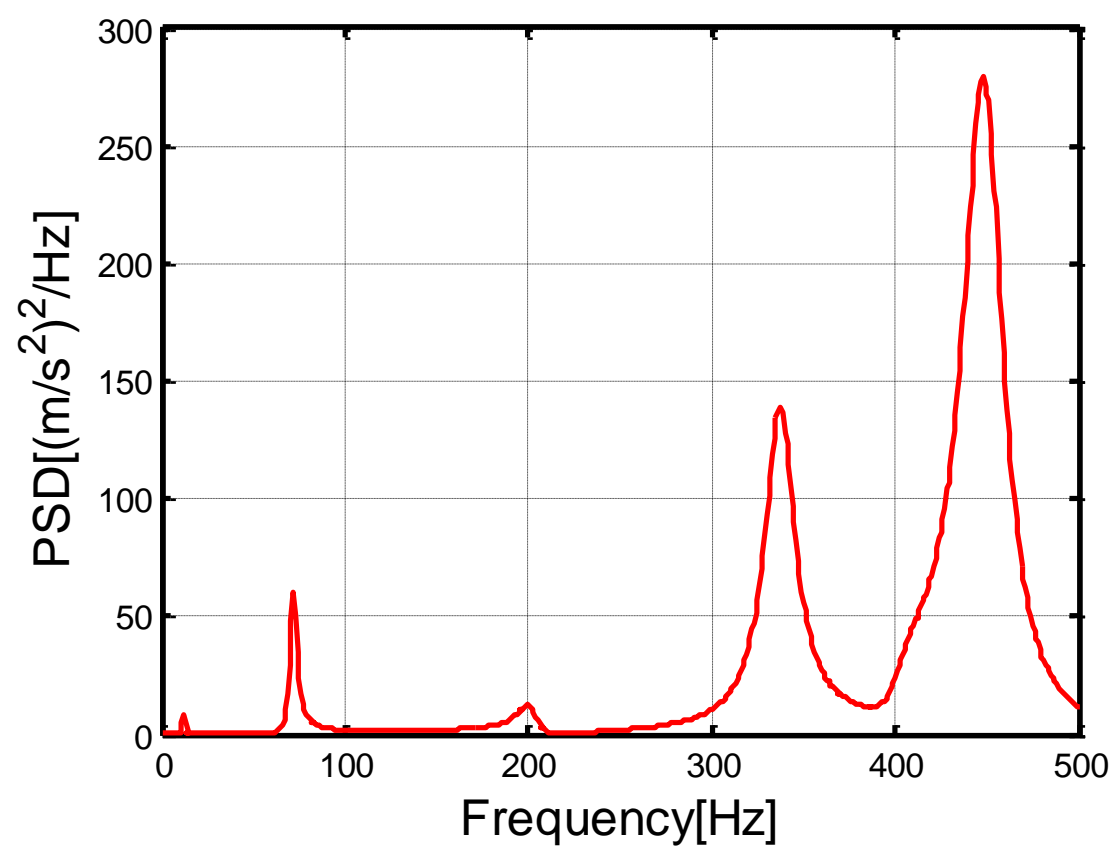

Fig. 4. Autopower spectral density function of the random dynamic acceleration response of response point 1 with $10 \%$ random noise.

To demonstrate the effectiveness and accuracy of the proposed modified regularization method, the modified regularization method presented in Section 3 and the Moore-Penrose inverse method as well as Tikhonov regularization method are applied to identify the random excitation with the simulated responses based on the cantilever plate. The results of the identified loads by different methods based on the autopower spectral density (Auto-PSD) and cross-power spectral density (Cross-PSD), are compared with the actual excitations as shown in Fig. 5(a-c) and Fig. 6(a-b). It is observed that the identified results by Moore-Penrose pseudo inverse method have huge errors in the vicinity of natural frequencies, especially in the low frequency region. Due to the relatively small condition number of FRF matrix in the other frequency range, the identified results by traditional Tikhonov regularization method are overly regularized leading to grievous inaccuracy of 
identification. On the contrary, the identified results by the modified regularization method reduce the error remarkably and enormously and match the actual excitation fairly well in the whole frequency region. As the modified regularization parameter is adjusted for each order pseudo excitation and condition number of FRF matrix at each frequency, it is able to ensure the stability of identified random excitation in the low frequency range and to prevent the identified results from over regularization under the circumstance of small condition number. As shown in the figures, MR denotes the modified regularization method, MPI represents the Moore-Penrose pseudo inverse method, and TR is the traditional Tikhonov regularization method.

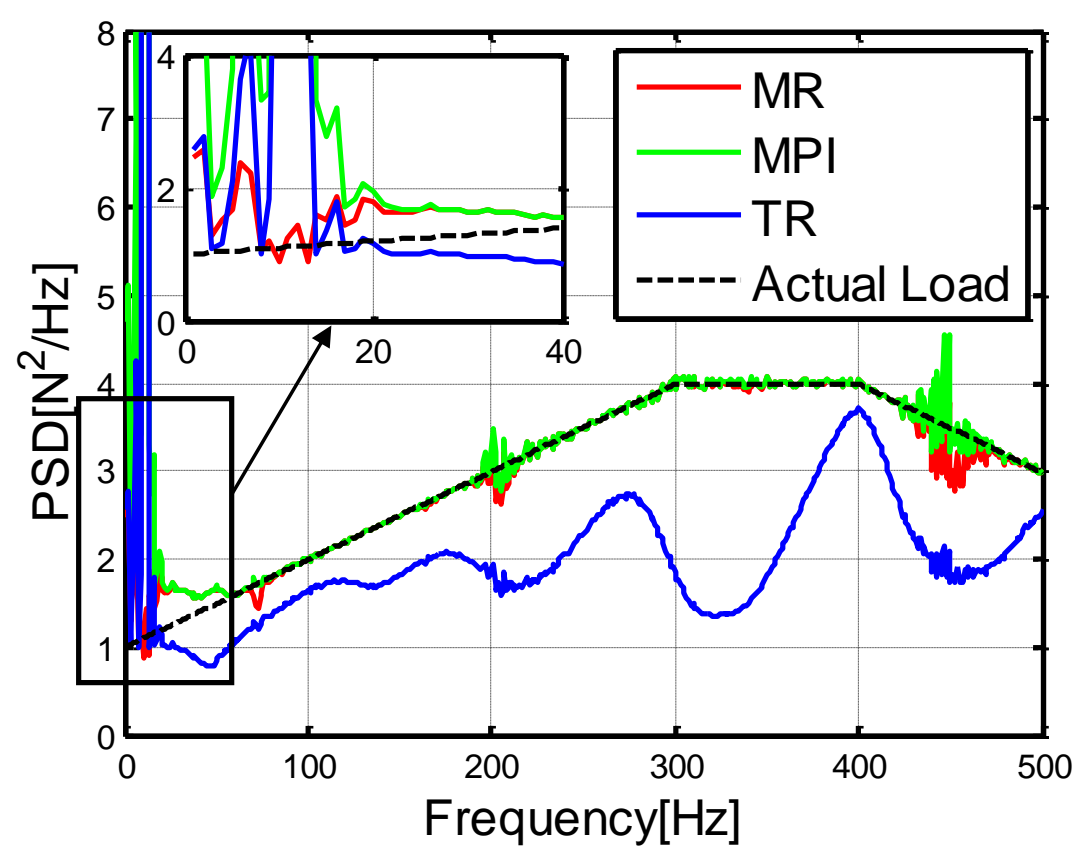

(a) 


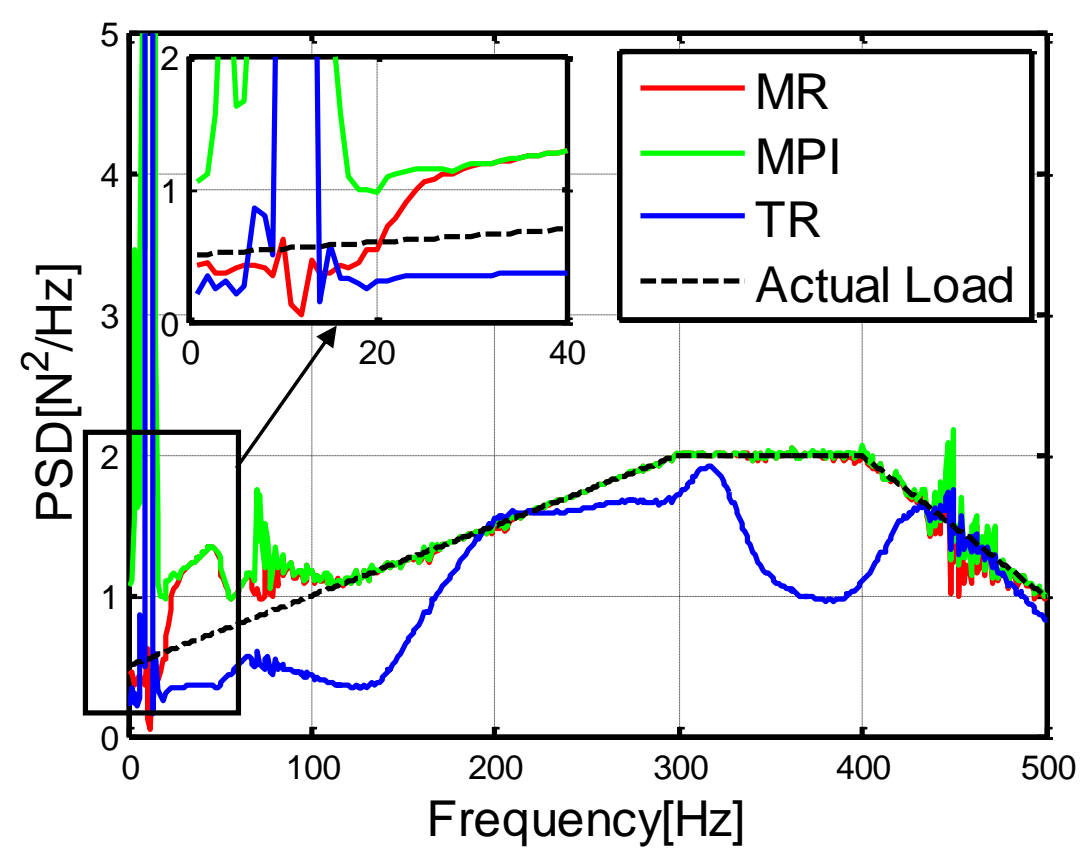

(b)

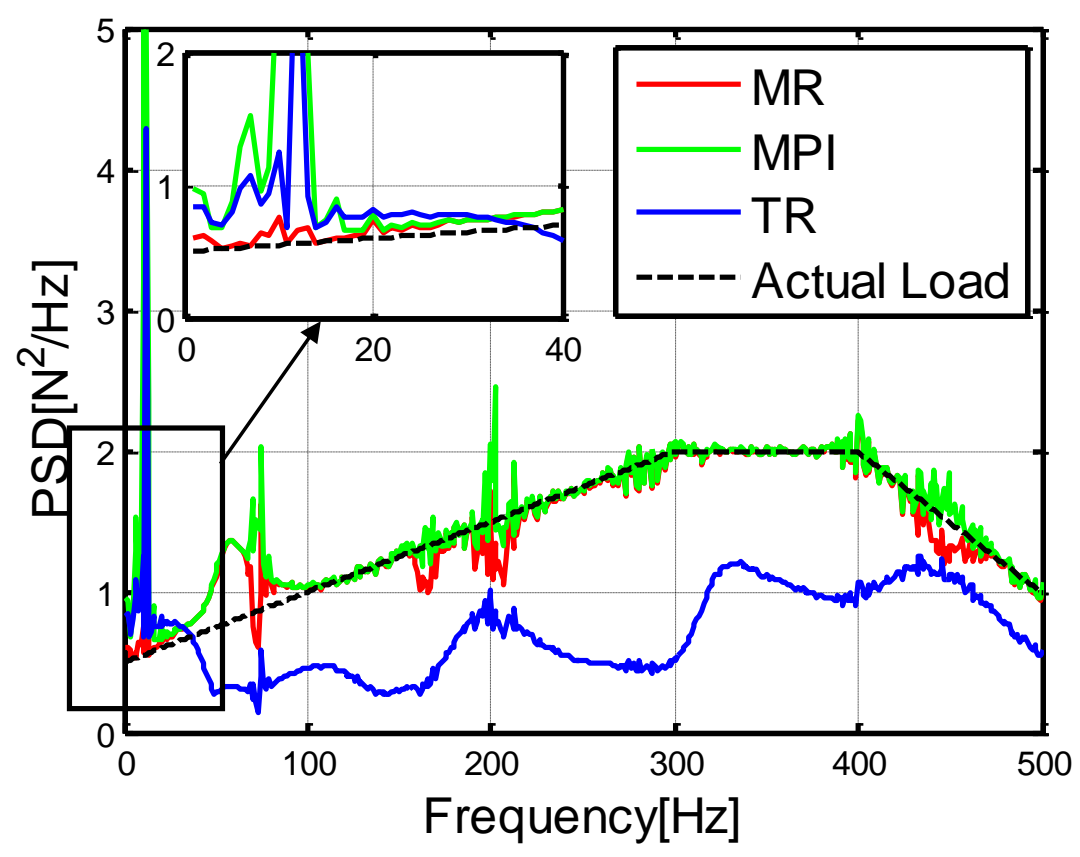

(c)

Fig. 5. Comparison of the Auto-PSD of identified load by modified regularization method and other two methods with the actual loads: (a) exciting point I; (b) exciting point II; (c) exciting point III. 


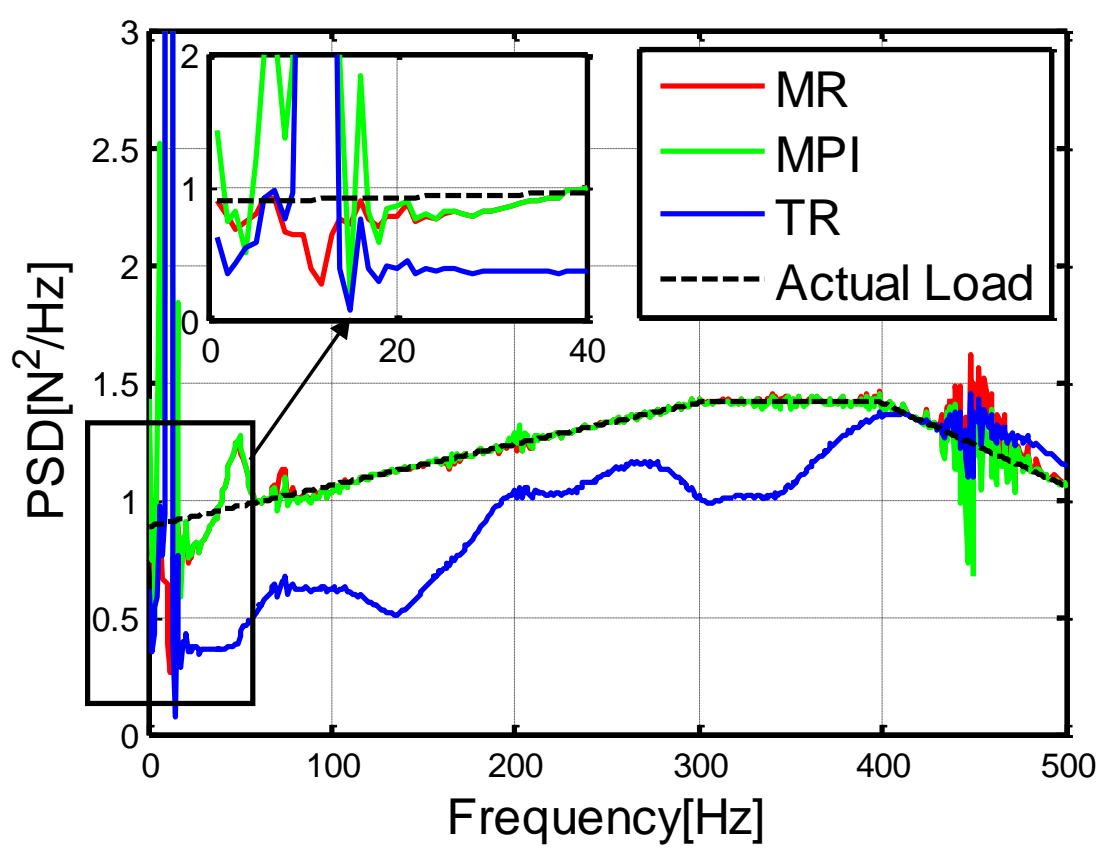

(a)

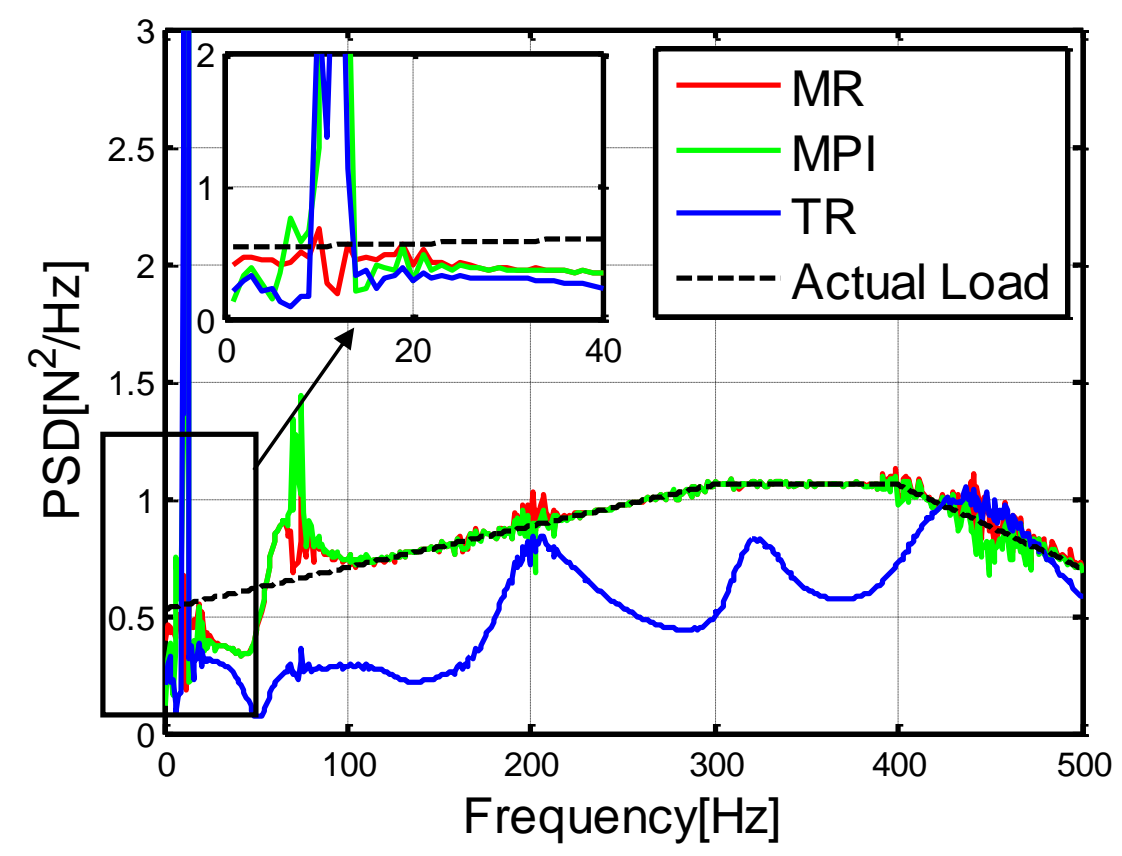

(b)

Fig. 6. Comparison of the Cross-PSD magnitude of identified load by modified regularization method and other two methods with the actual loads: (a) between exciting point I and exciting point II;

(b) between exciting point II and exciting point III. 
Additionally, to further reflect the superiority and excellent performance of the modified regularization compared with traditional random excitation identification methods, the proposed method is applied on the model with the worst measurement locations. And the identified results shown in Fig. 7(a-c) are compared with the results by means of the rest of identification methods based on the best measurement locations. As seen in the identified results, compared with the Moore-Penrose pseudo inverse method, the modified regularization method is able to greatly solve the ill-posed problem and reduce the identified error in the low frequency range, even though it employs the worst measurement locations, and it can prevent the identified results from regularizing overly, which exists in the results by traditional Tikhonov regularization method. Obviously, it is obligatory to measure much more responses data for selecting the best measurement locations, but in the practical engineering application, it would increase the difficulty of testing data and require more testing time. Therefore, it can be deduced that the proposed method is superior to other methods because it is able to obtain acceptable identified results even without selecting the best measurement locations.

To conclude, compared with the Moore-Penrose pseudo method and traditional Tikhonov regularization method, the proposed modified regularization method can effectively and prominently reduce the identification error and improve the accuracy as well as the stability of the identified results. 


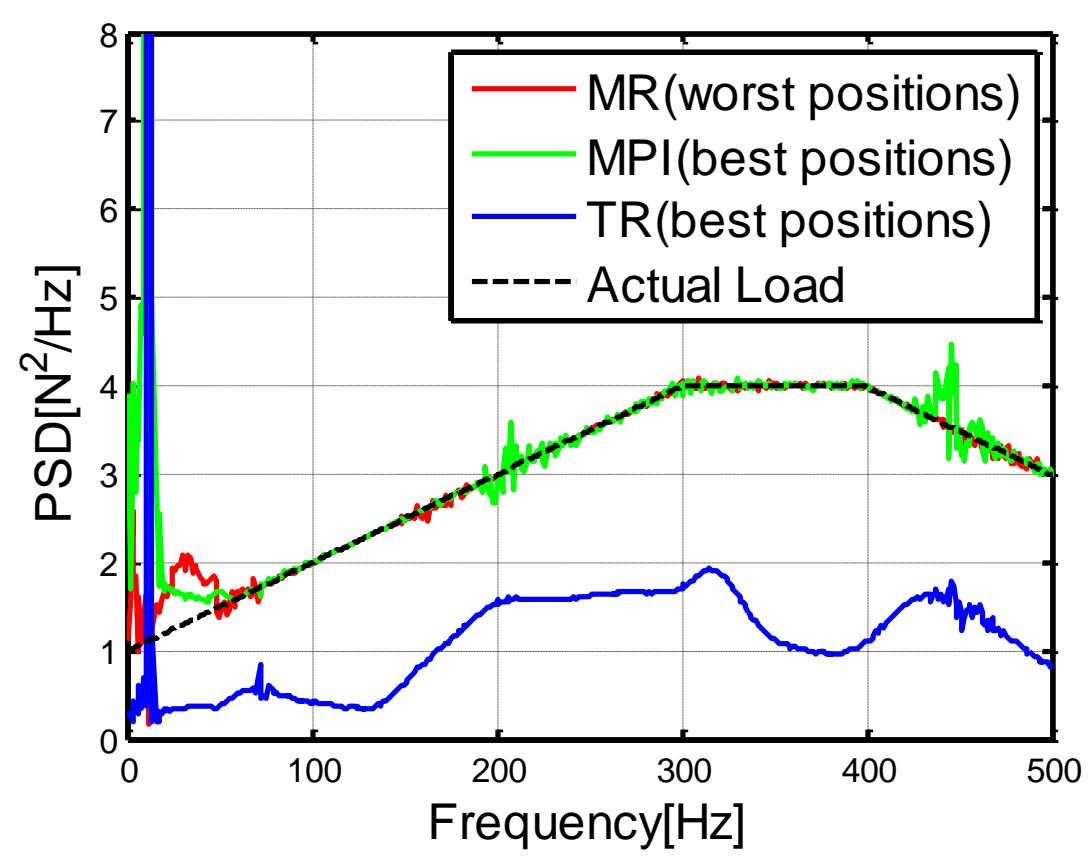

(a)

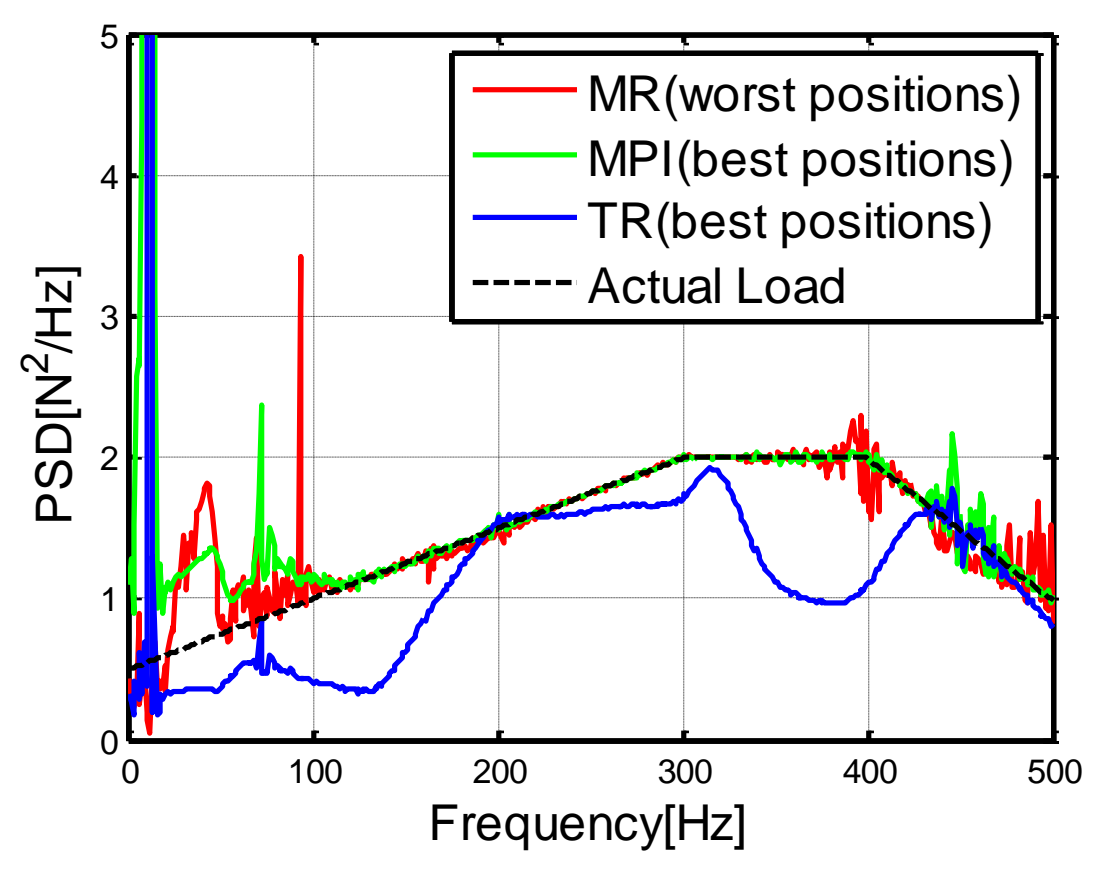

(b) 


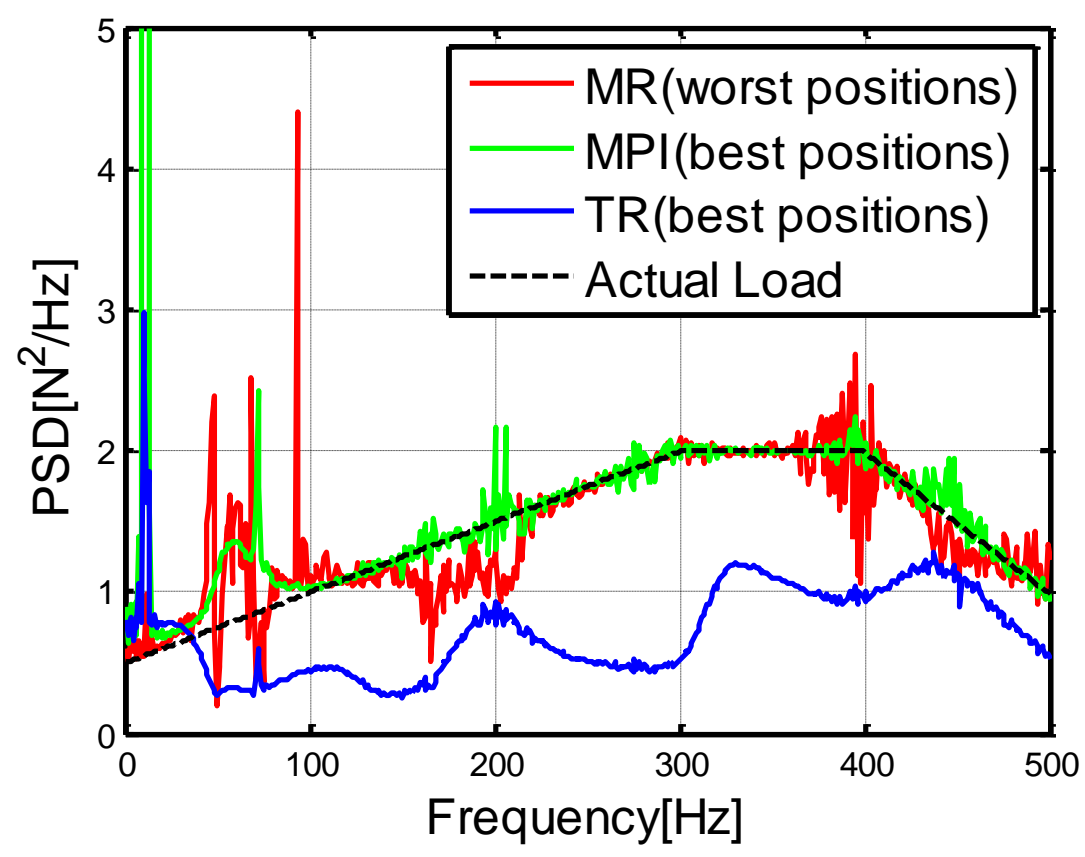

(c)

Fig. 7. Comparison of the Auto-PSD of identified load by modified regularization method on worst measurement locations and other two methods on best measurement locations with the actual loads: (a) exciting point I; (b) exciting point II; (c) exciting point III.

\subsubsection{Simulation-based validation for perturbation method}

The numerical example investigated in the above section is the identification of random dynamic excitation for deterministic structure. Considering the random structural parameters, we assume that the elastic modulus and material density of the candidate plate are uncorrelated random parameters following normal distribution due to the manufacture errors. The standard deviations of the elastic modulus and material density are $0.35 \mathrm{GPa}$ and $13.5 \mathrm{~kg} / \mathrm{m}^{3}$ respectively.

In the previous section, the mean value of pseudo excitation has been obtained by means of the modified regularization method, and the sensitivities of pseudo excitation with 
respect to the random parameters are calculated as shown in Fig. 8(a-b). Next, the sensitivities of random excitation PSD with respect to the random parameters are obtained, and the results are drawn in Fig. 9(a-b). Finally, the statistical characteristics of the identified random excitation, namely the bounds shown in Fig. 10(a-c) are derived. It is noted that the actual loads are mostly located within the bounds, thus the proposed method is proved to be effective and feasible in practical engineering circumstance. In addition, the bounds based on the mean value by Moore-Penrose inverse method and Tikhonov regularization method are also calculated as shown in Fig. 11(a-b). Clearly, due to the ill-posed and unstable solutions on the mean value of random parameters, the identified bounds by those two methods retain the huge error and deviate from actual load badly.

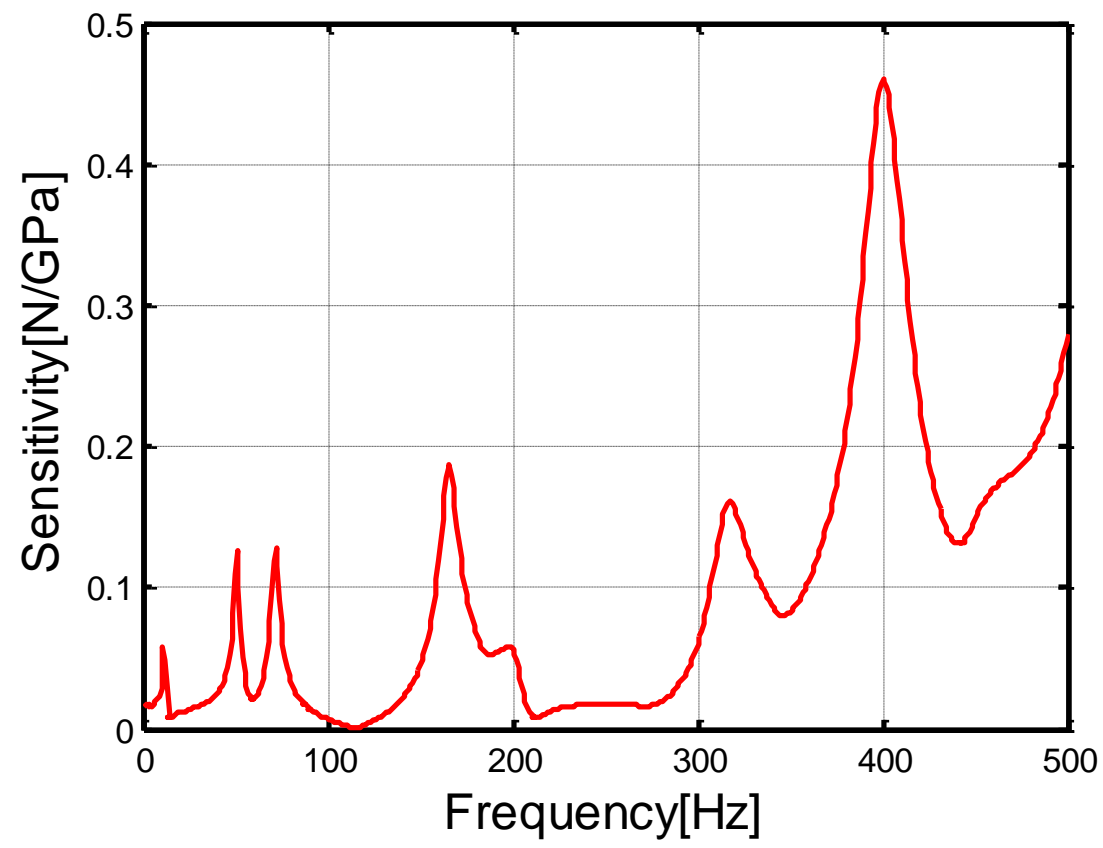

(a) 


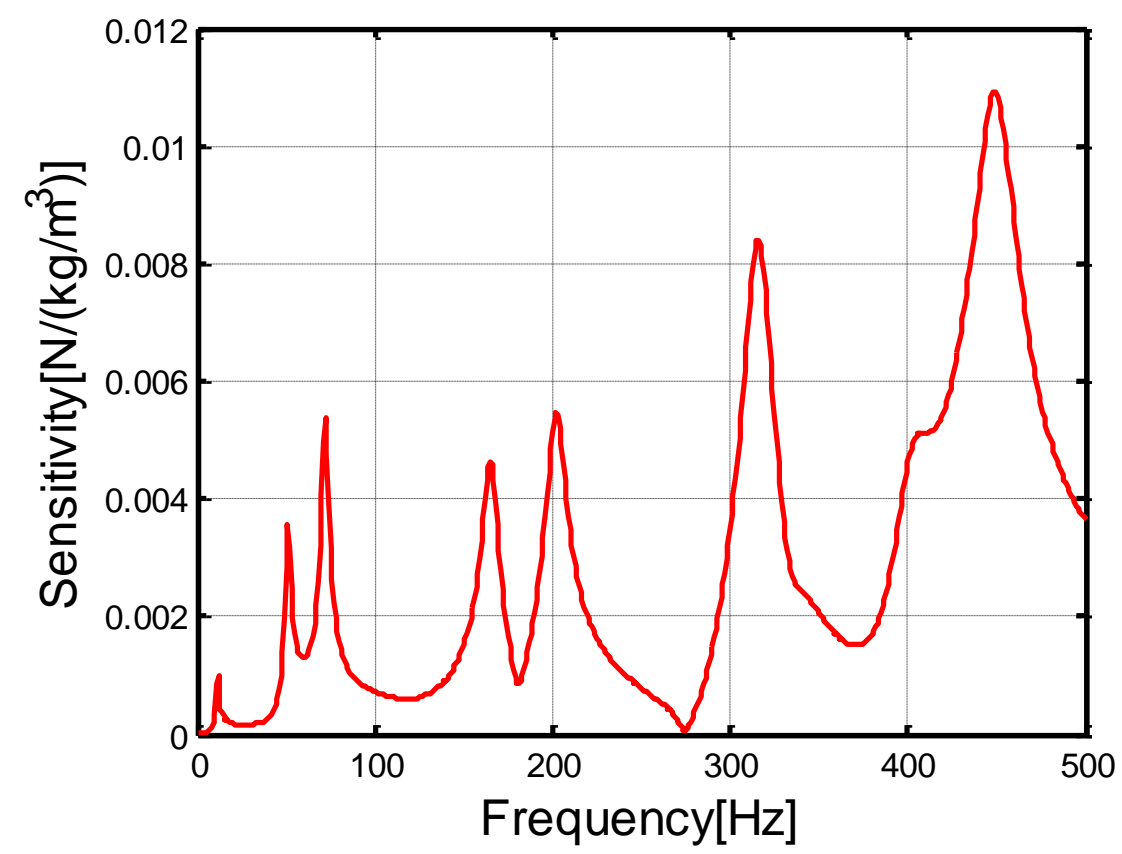

(b)

Fig. 8. (a) Sensitivity of first order pseudo excitation of exciting point I to elastic modulus;

(b) Sensitivity of first order pseudo excitation of exciting point II to material density.

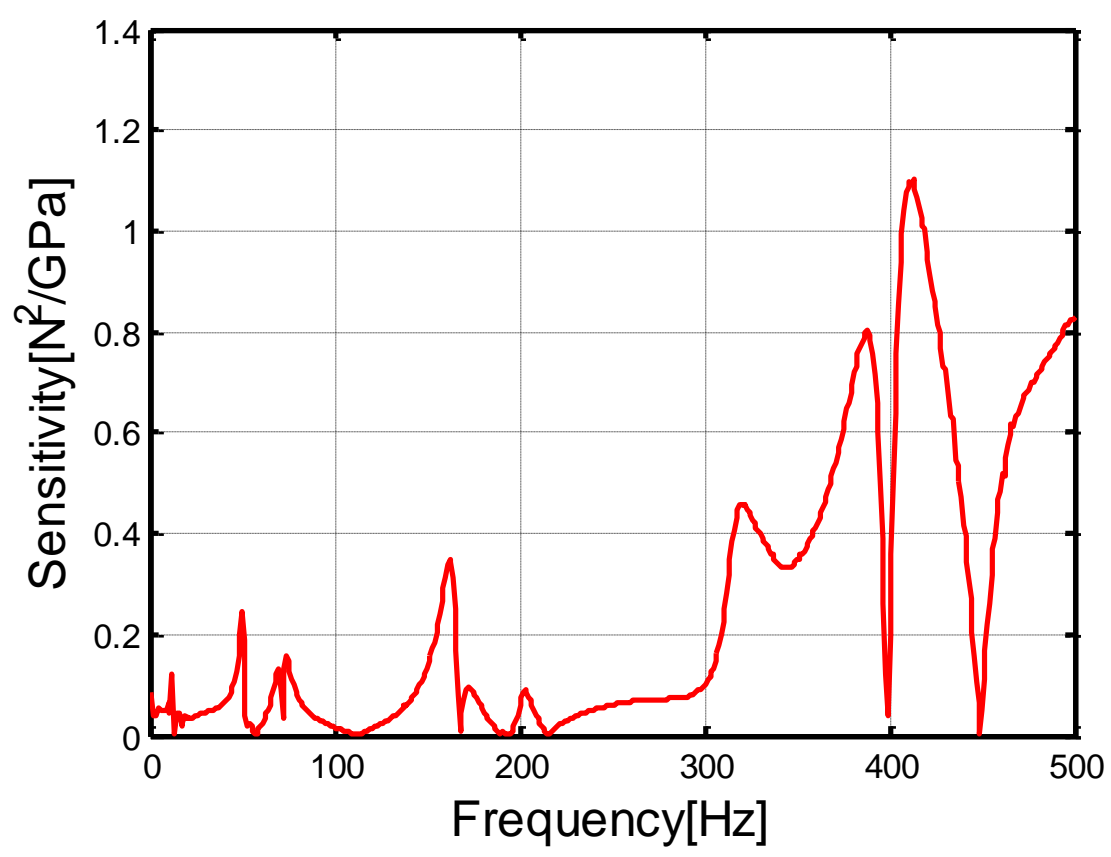

(a) 


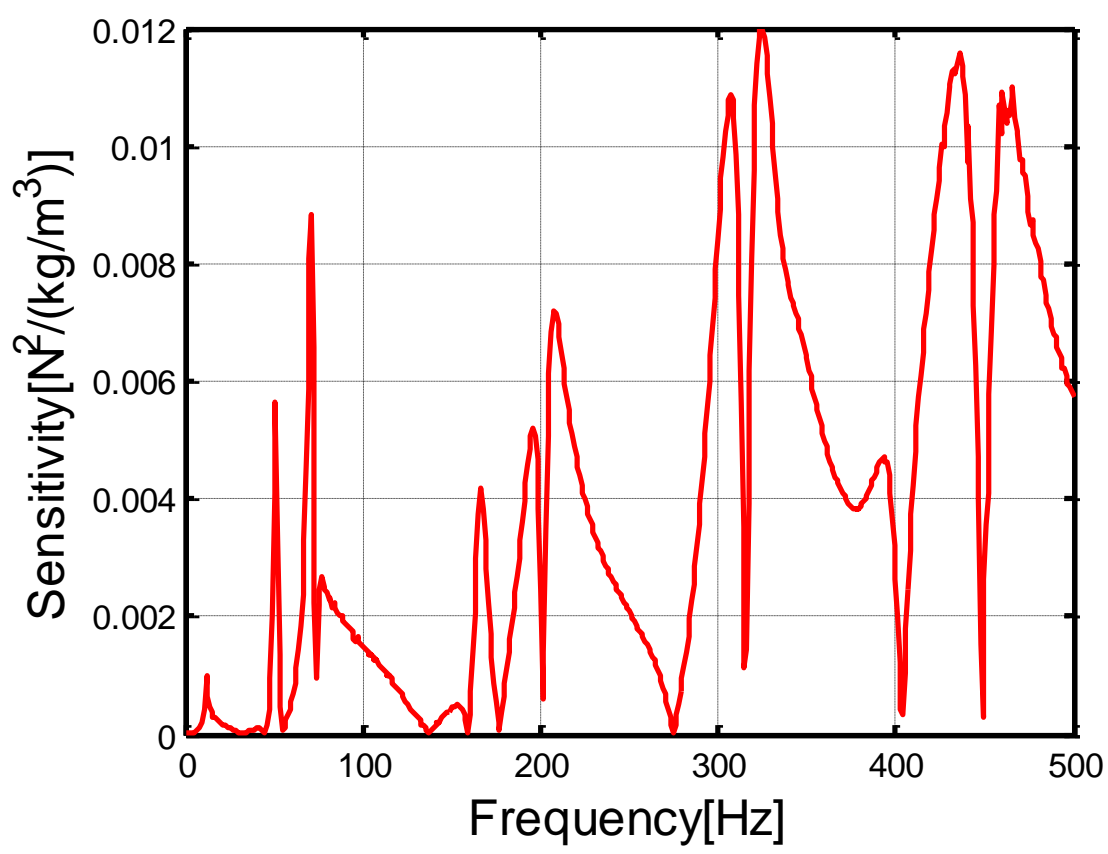

(b)

Fig. 9. (a) Sensitivity of the Auto-PSD of exciting point I to elastic modulus;

(b) Sensitivity of the Auto-PSD of exciting point II to material density.

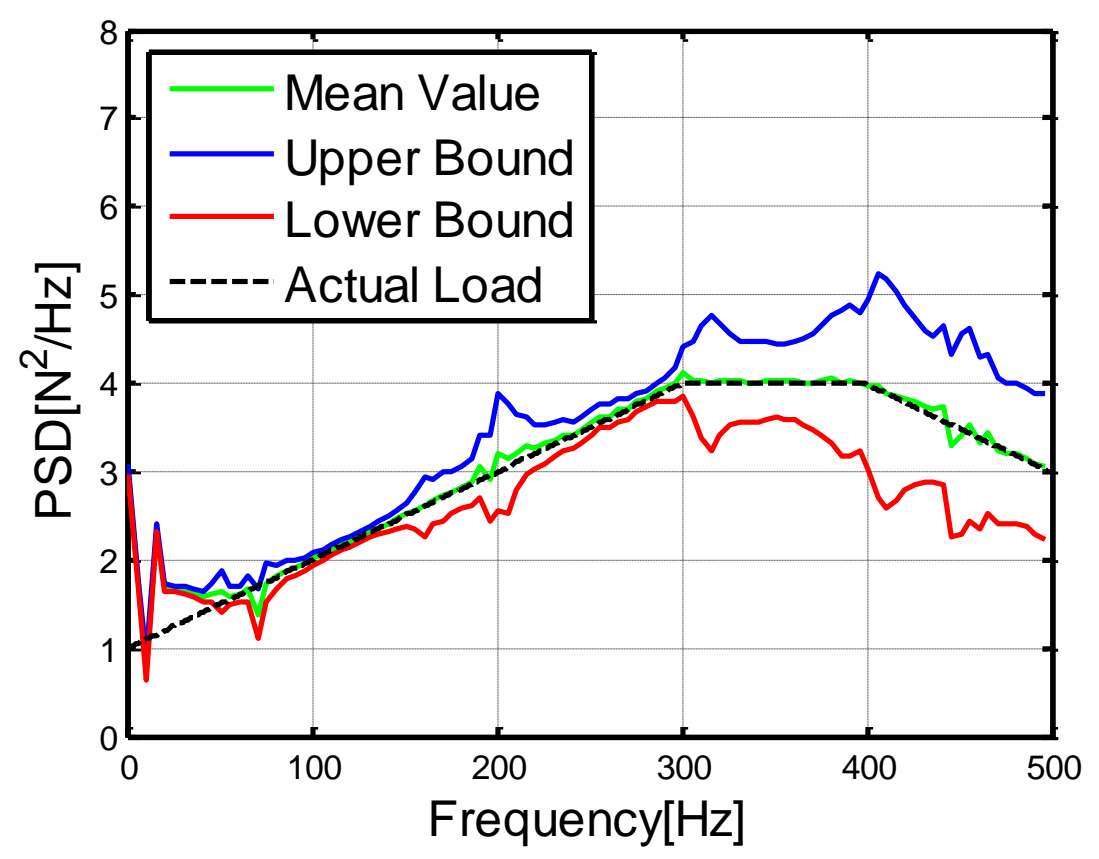


(a)

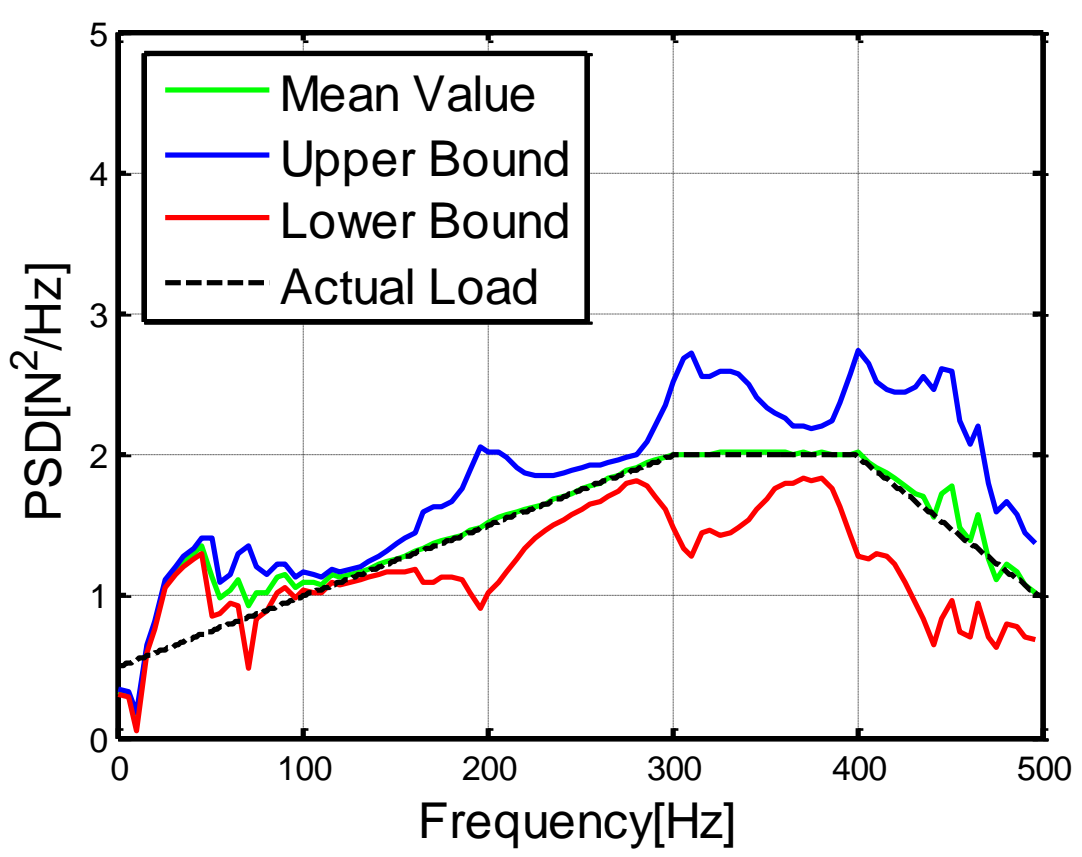

(b)

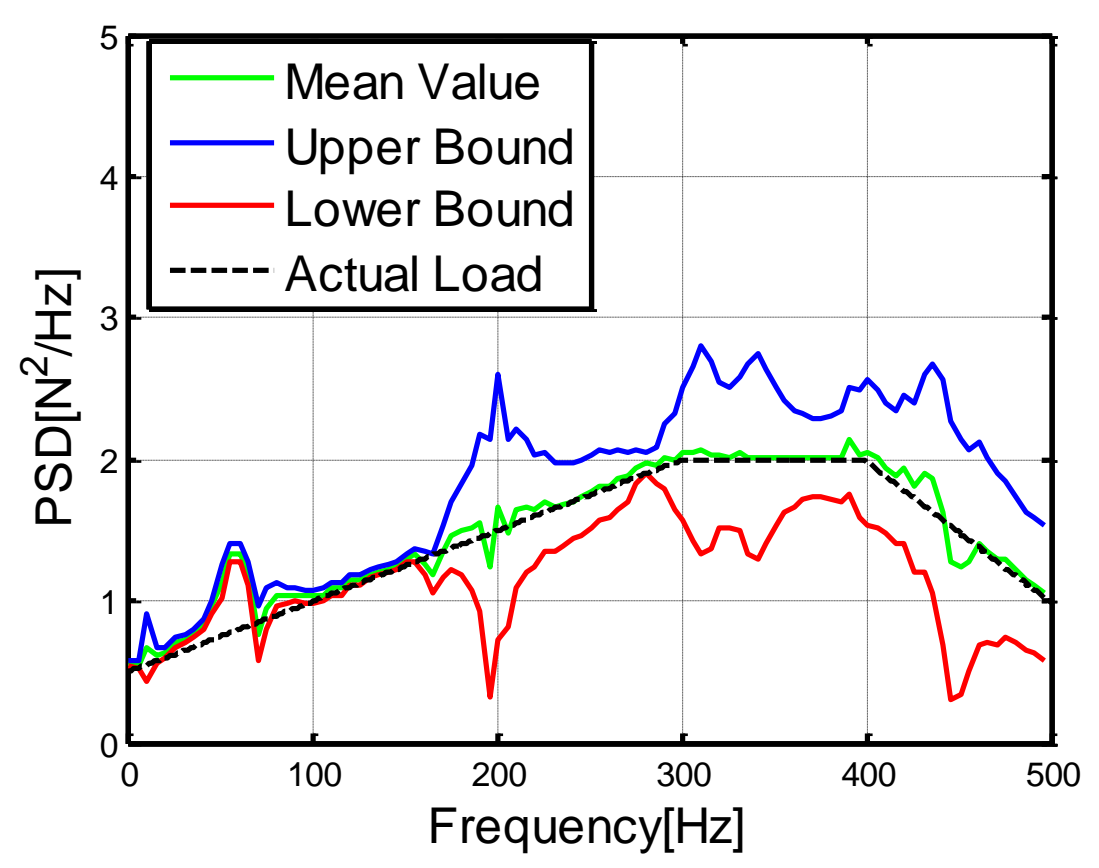

(c)

Fig. 10. The bounds of Auto-PSD of the identified random excitation (a) exciting point I; (b) exciting point II; (c) exciting point III. 


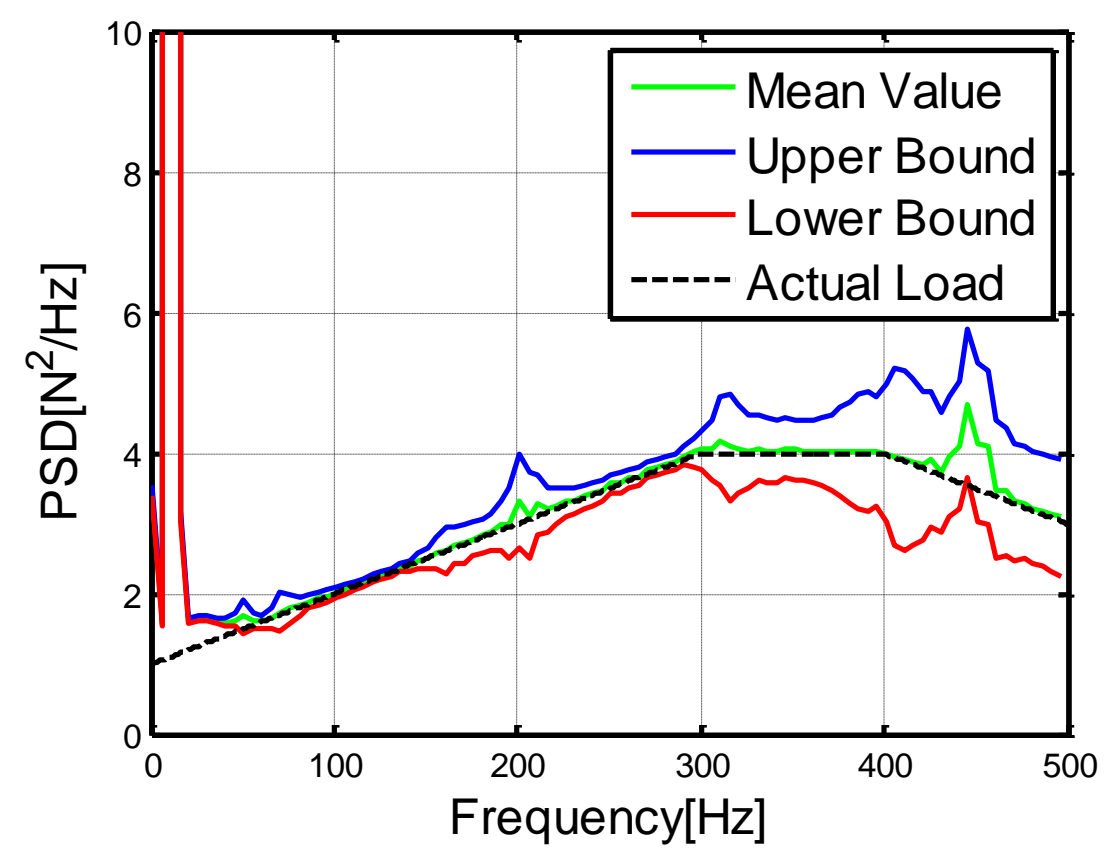

(a)

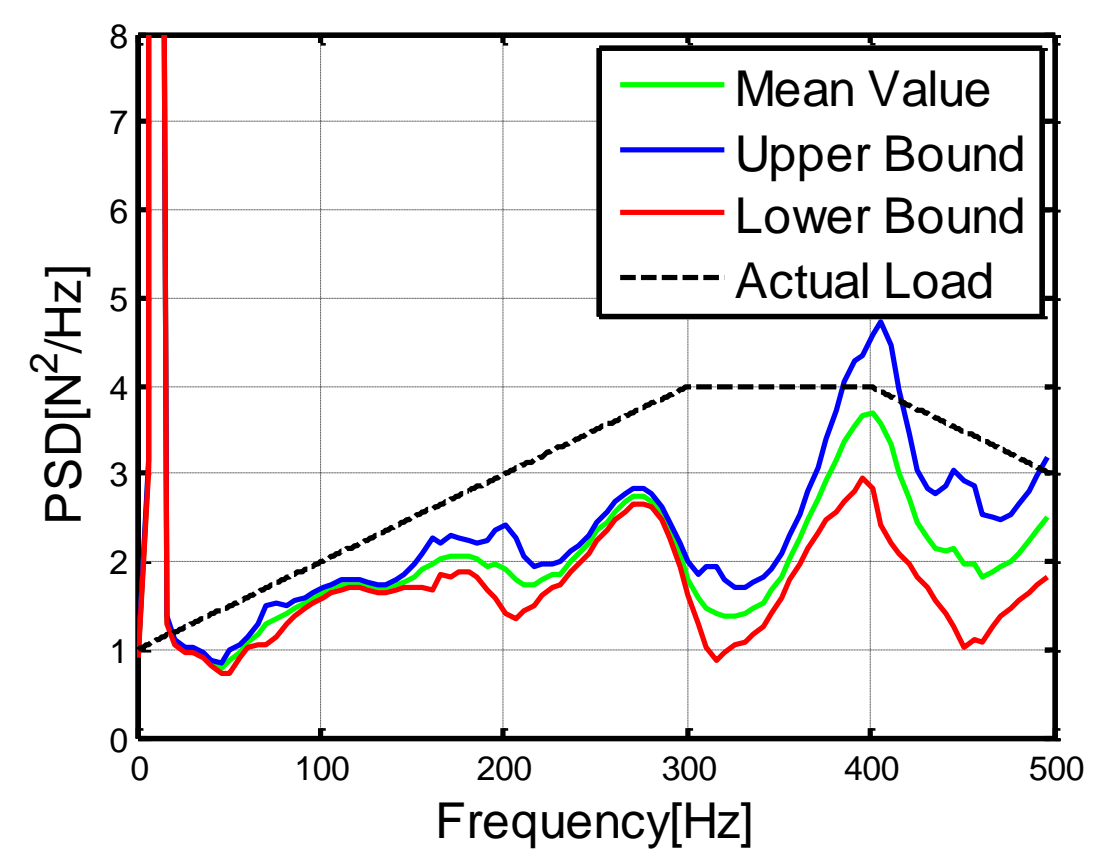

(b)

Fig. 11. The bounds of Auto-PSD of the identified random excitation at exciting point I by other two methods (a) Moore-Penrose pseudo inverse; (b) Tikhonov regularization. 


\subsection{A vehicle door problem}

The vehicle door plays an important role in the overall design of a vehicle, and the dynamic characteristics of the door have a strong effect on the safety of passengers. The technology of dynamic load identification can be used for investigating the impact of extra forces on the vehicle door and early design of the door. The simplified vehicle door as shown in Fig.12 is mainly made up of inner plate, exterior plate, glass, and several pieces of vertical and longitudinal stiffener plates. The material densities of the door plates and the window glass are $7850 \mathrm{~kg} / \mathrm{m}^{3}$ and $2500 \mathrm{~kg} / \mathrm{m}^{3}$ respectively, the elastic modulus are 205 GPa and $69 \mathrm{GPa}$ respectively, the Poisson's ratios are 0.30 and 0.29 respectively, and the structural damping loss factors are both 0.06. Point A and B are fixed. Two exciting points and eight candidates for responses points are selected in $y$-direction randomly. We assume that the elastic modulus of exterior plate and material density of inner plate and exterior plate are mutually uncorrelated random parameters obeying Gaussian distribution. The standard deviations of the elastic modulus and material density are $2.05 \mathrm{GPa}, 78.5 \mathrm{~kg} / \mathrm{m}^{3}$ and $78.5 \mathrm{~kg} / \mathrm{m}^{3}$ respectively.

Based on the method of composite condition number, the best combination of four measurement locations from eight ones are obtained, which is $1,3,4,6$. The comparison of the FRF matrix condition number between the best combination and worst one is shown in Fig.13. After that, when the random parameters take the mean value, the random dynamic acceleration responses are obtained through the given constructed random excitations, moreover, a noise is added to the computational responses as shown in Fig.14. 

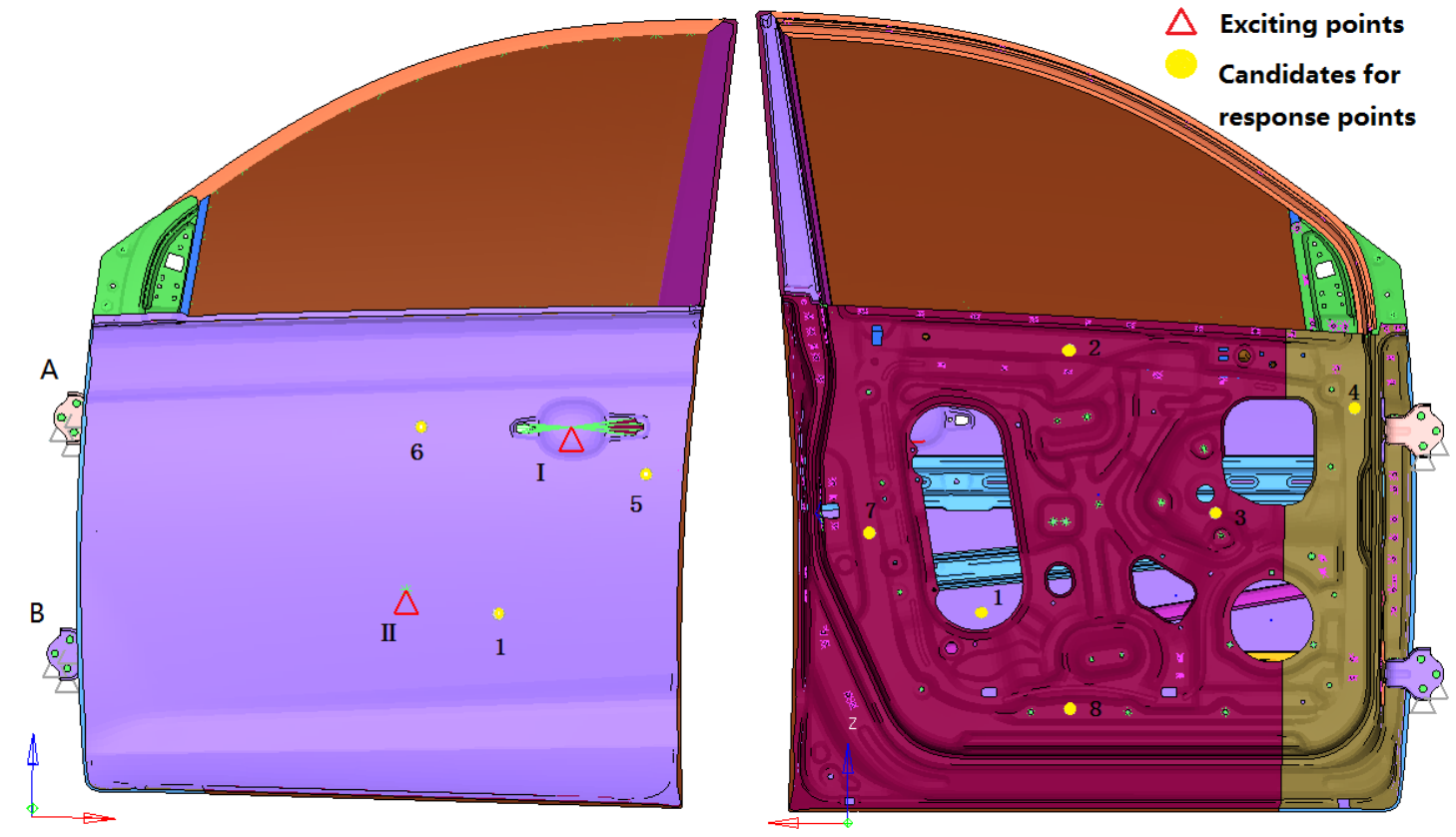

Fig. 12. The finite element model of vehicle front door.

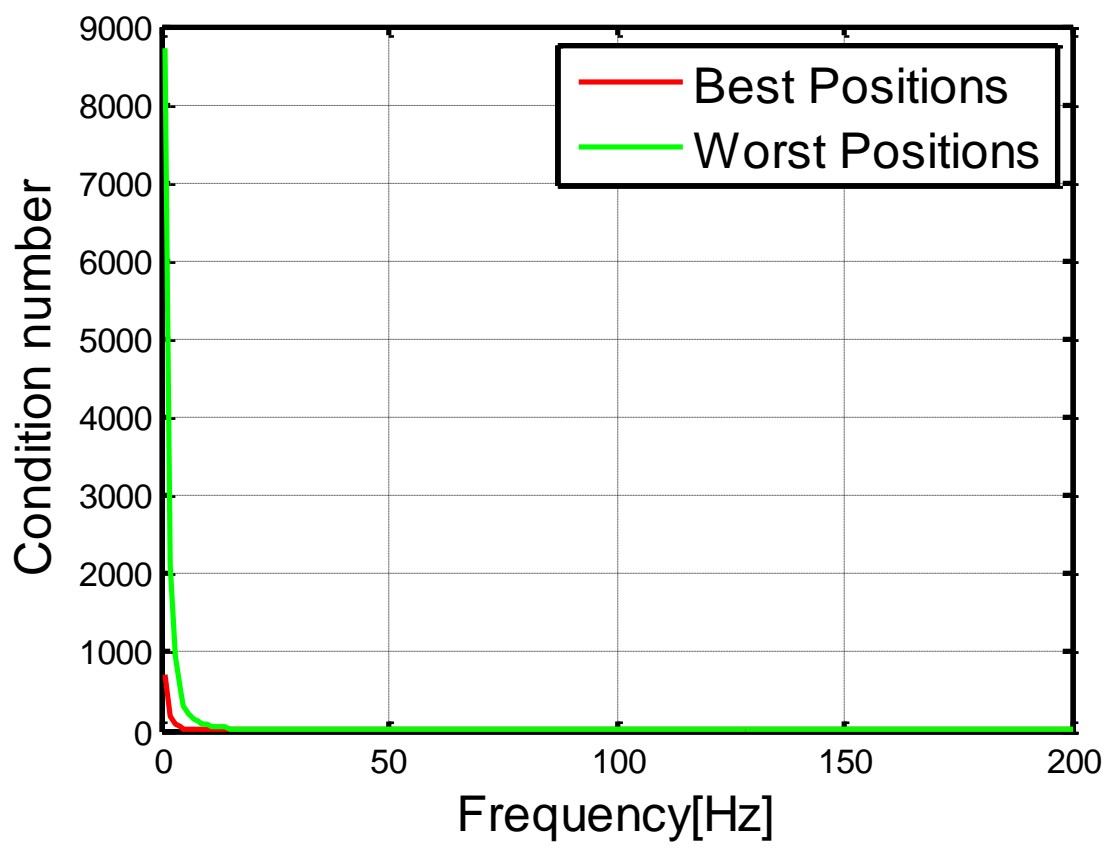

Fig. 13. Comparison of the FRF matrix condition number between best combination of measuring locations and worst one. 


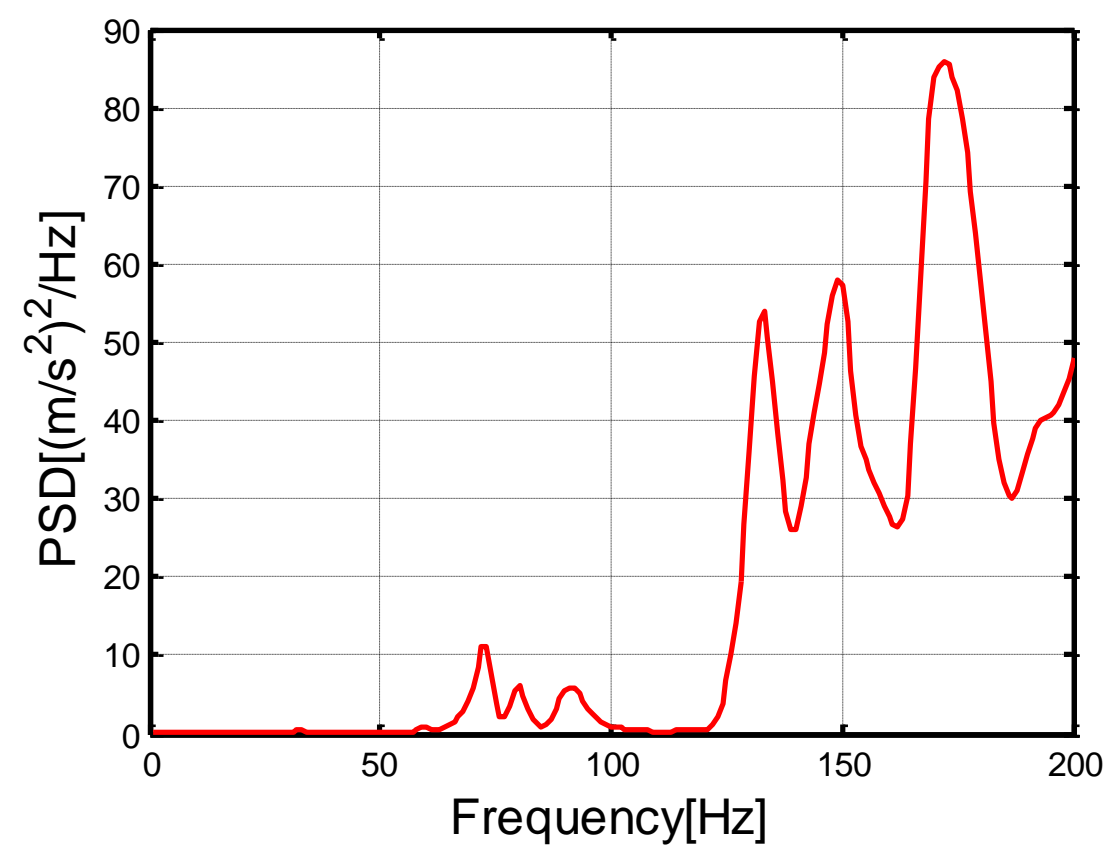

Fig. 14. Auto-PSD function of the random dynamic acceleration response of point 1 with $5 \%$ random noise.

The results of the identified loads by modified regularization method and other two identified methods are compared with the actual excitations as shown in Fig. 15 (a-c). It can be found that the modified regularization method is able to reduce the identified error in the low frequency range and prevent the identified results from regularizing overly. In addition, it is still observed that the gaps between the identified loads from traditional Tikhonov regularization method and the actual loads are very large. Although the Moore-Penrose pseudo inverse method can provide reasonable results in the high frequency range, the identified loads from Moore-Penrose pseudo inverse method are still poor in the low frequency range. Thus, the effectiveness and applicability of the proposed modified regularization method have been demonstrated clearly.

Through computing the sensitivities of pseudo excitation and random excitation with regard to random structural parameters shown in Fig. 16(a-c) and Fig. 17(a-c), the bounds of 
the identified random excitation are derived as shown in Fig. 18(a-b). It can be seen that the bounds mostly bracket the actual excitation. Therefore, the matrix perturbation method can provide stable and effective bounds of identified dynamic loads in practical engineering application.

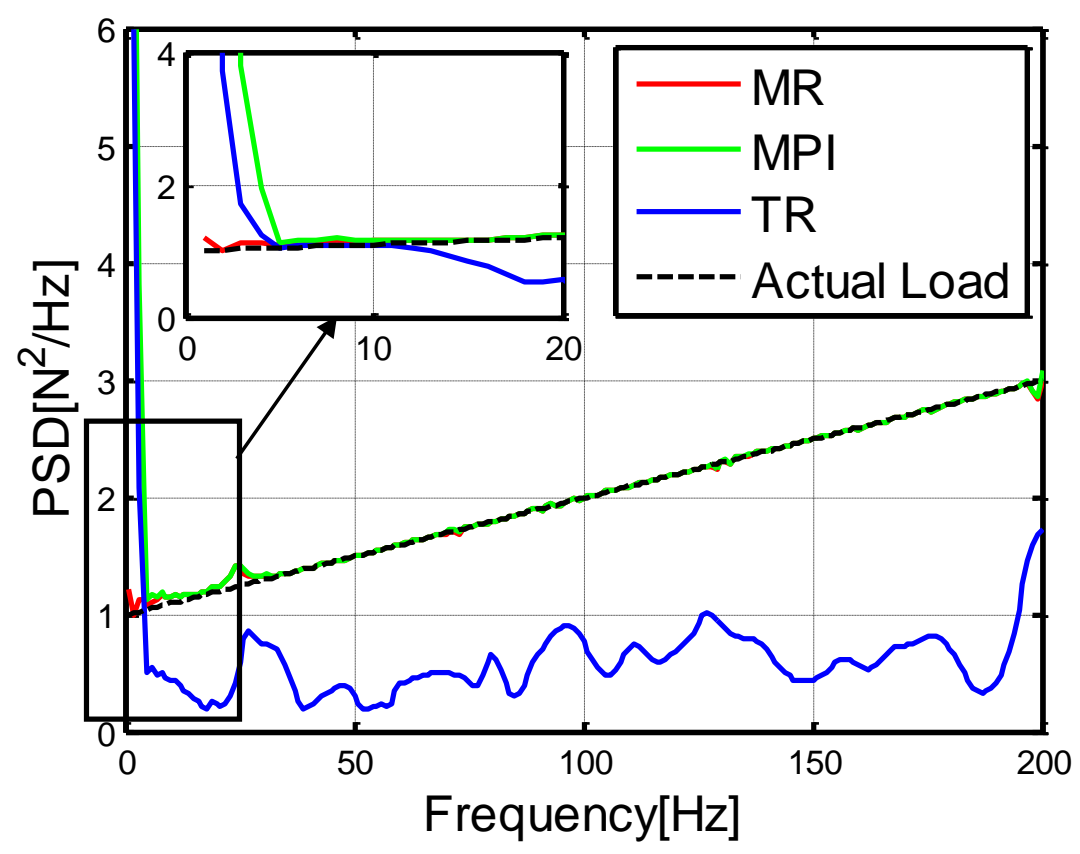

(a) 


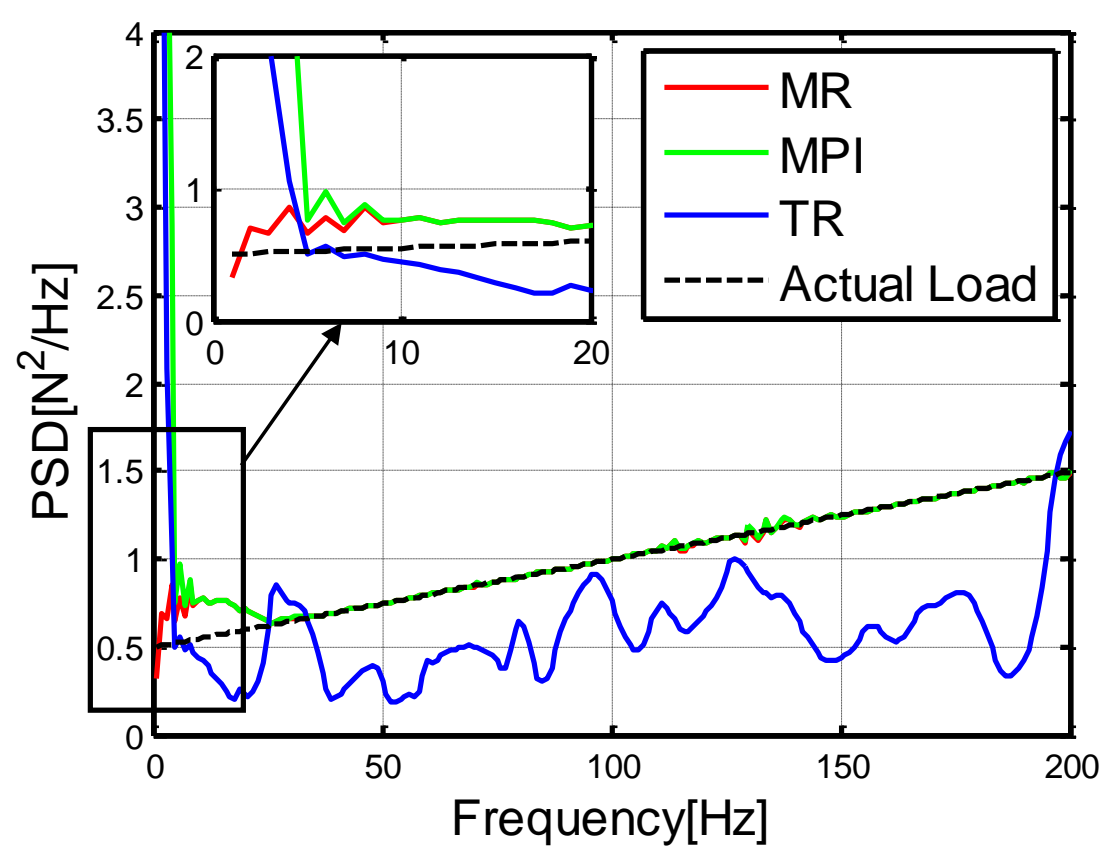

(b)

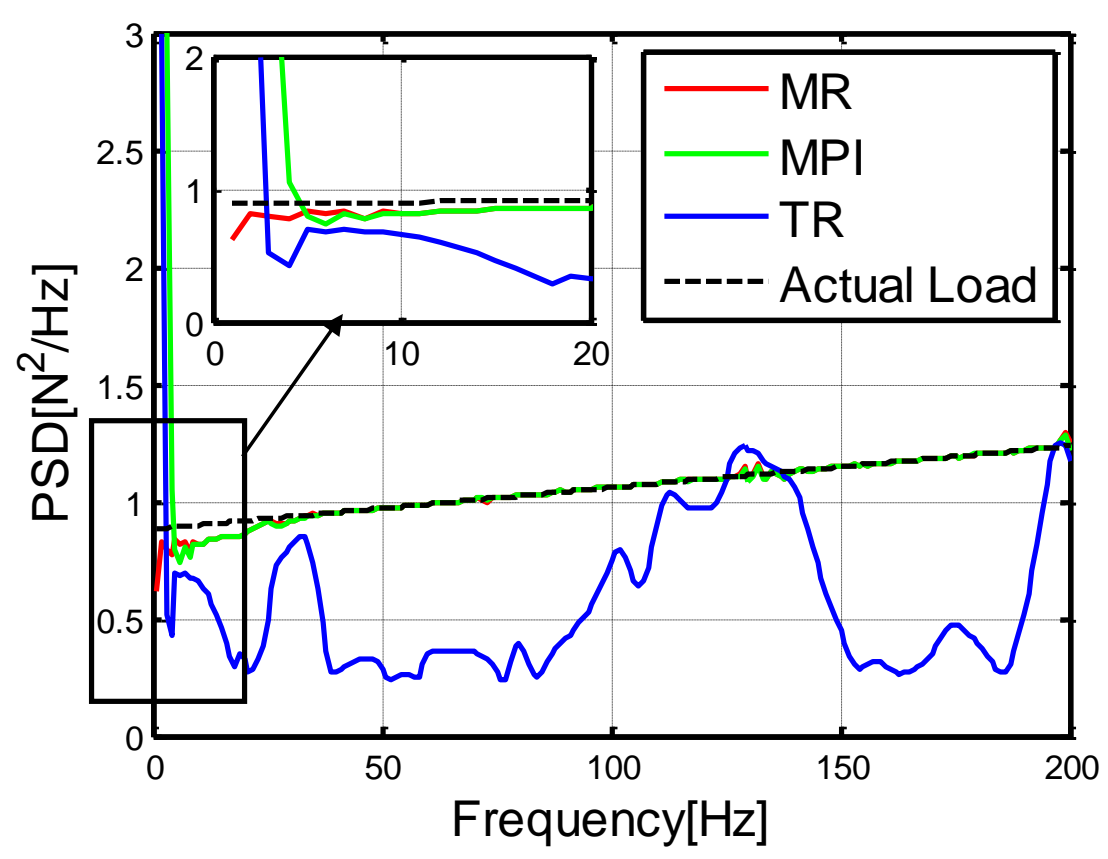

(c)

Fig. 15. Comparison of the identified load by modified regularization method and other two methods with the actual loads: (a) Auto-PSD of exciting point I; (b) Auto-PSD of exciting point II; (c) Cross-PSD between exciting point I and exciting point II. 


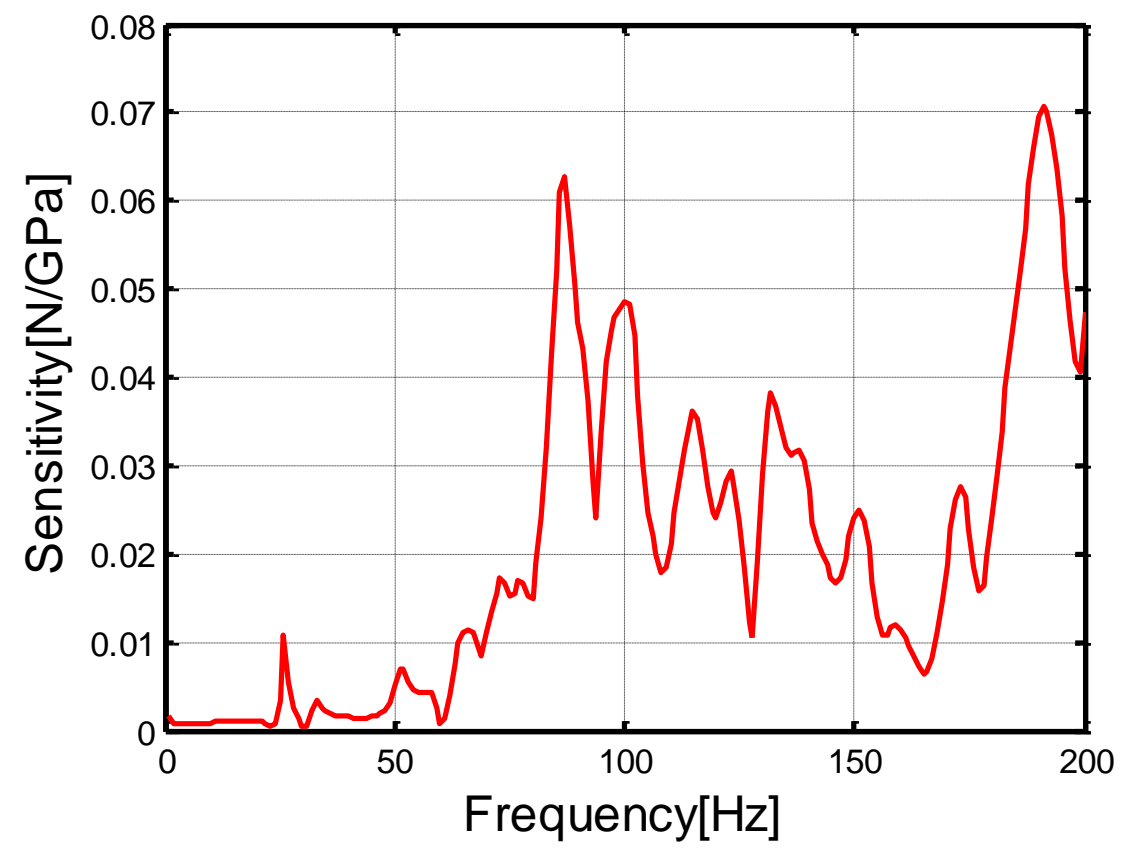

(a)

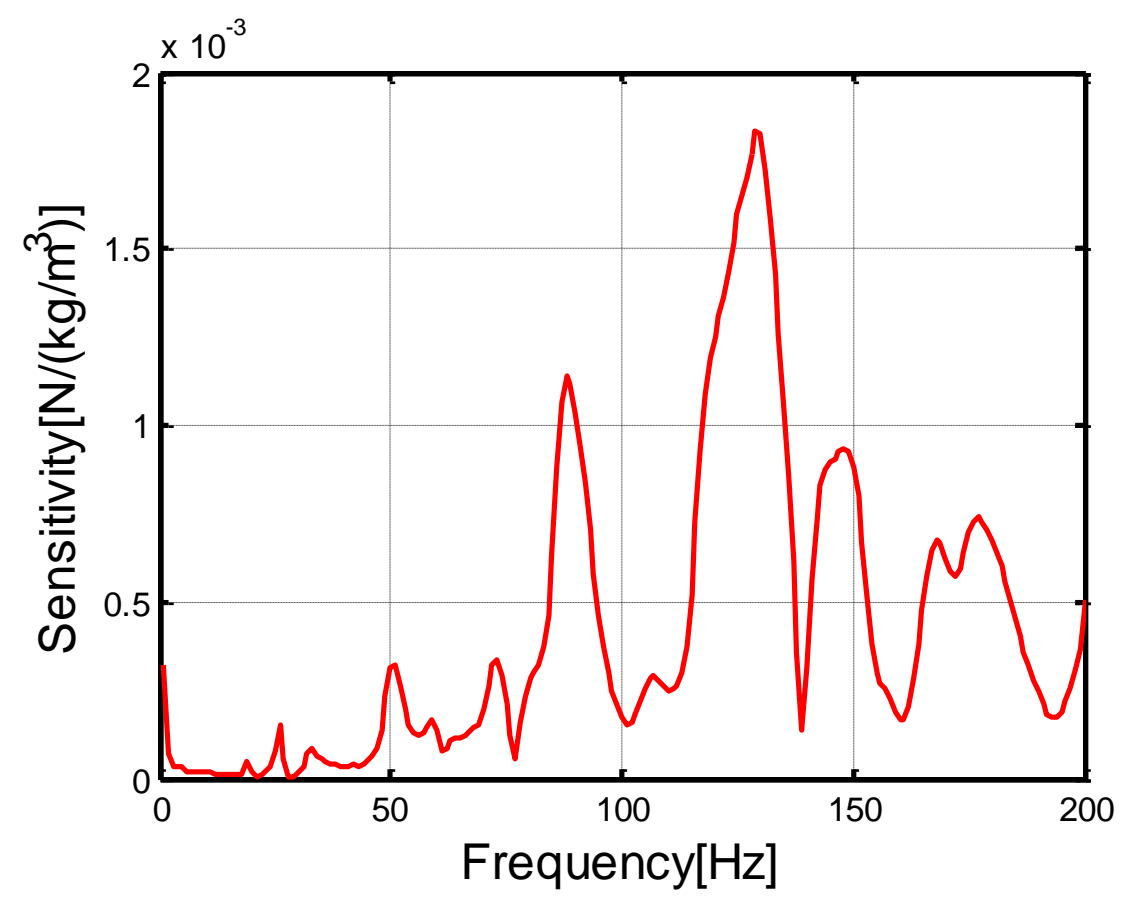

(b) 


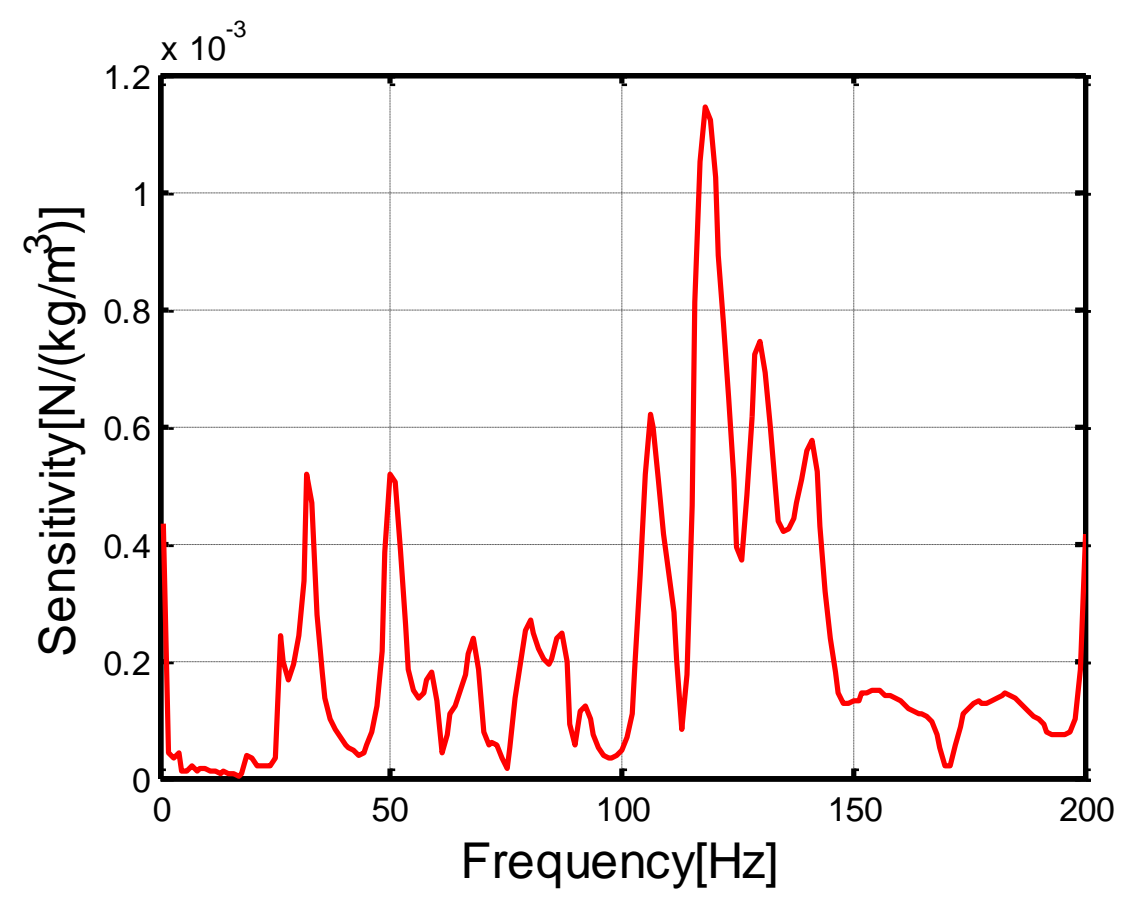

(c)

Fig. 16. (a) Sensitivity of first order pseudo excitation of exciting point I to elastic modulus of exterior plate; (b) Sensitivity of first order pseudo excitation of exciting point II to material density of exterior plate; (c) Sensitivity of first order pseudo excitation of exciting point II to material density of inner plate.

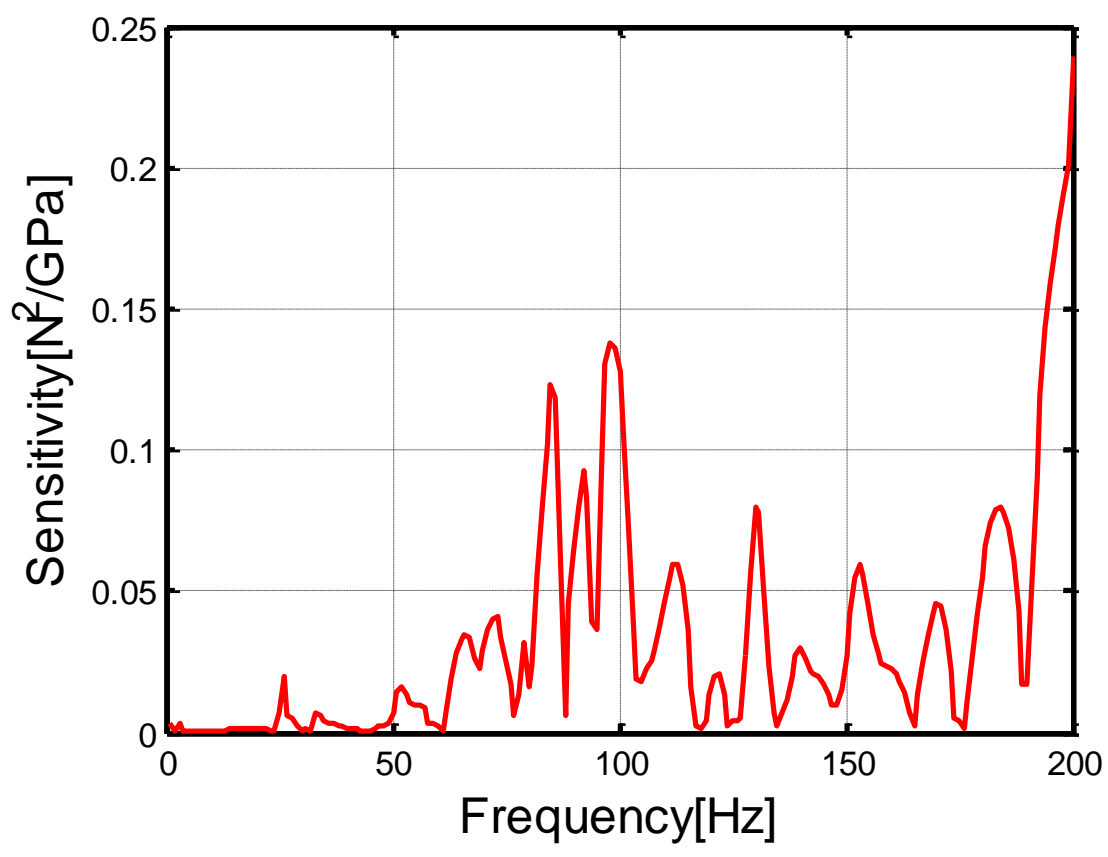

(a) 


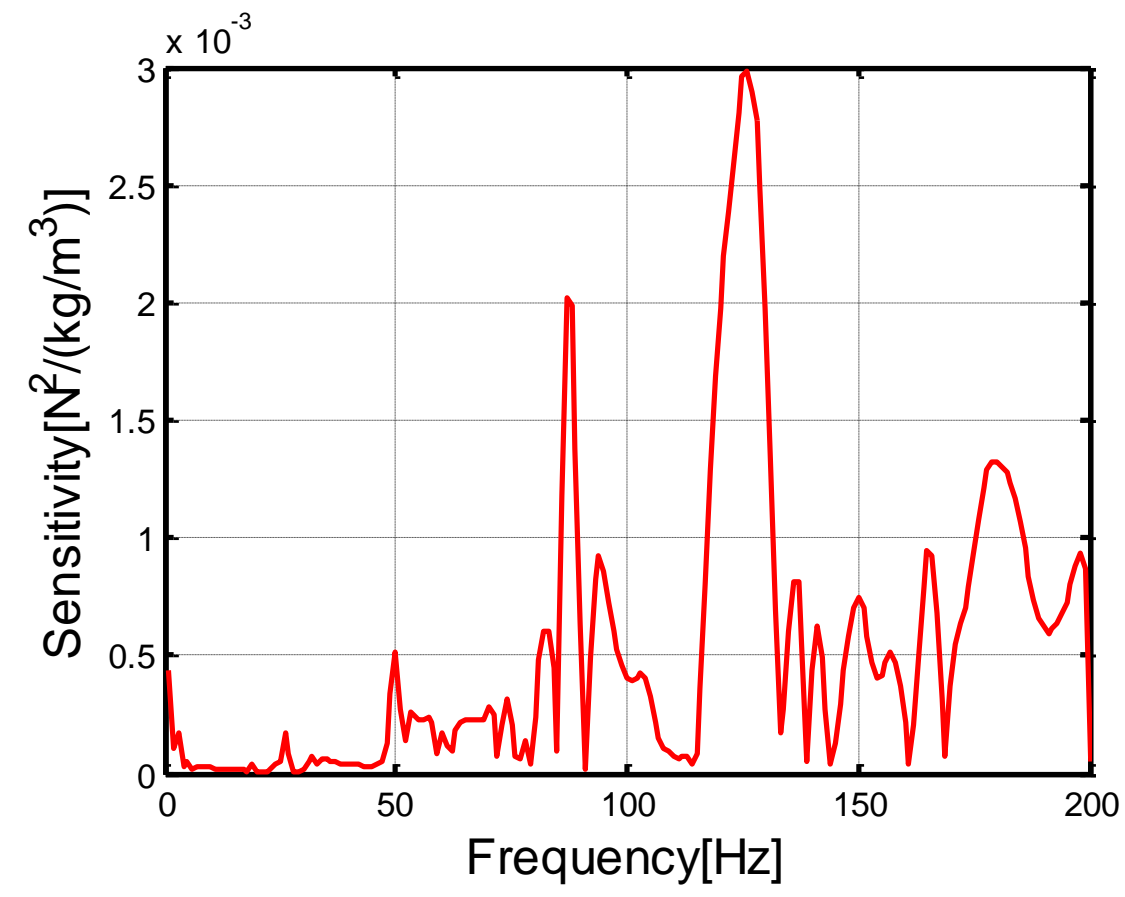

(b)

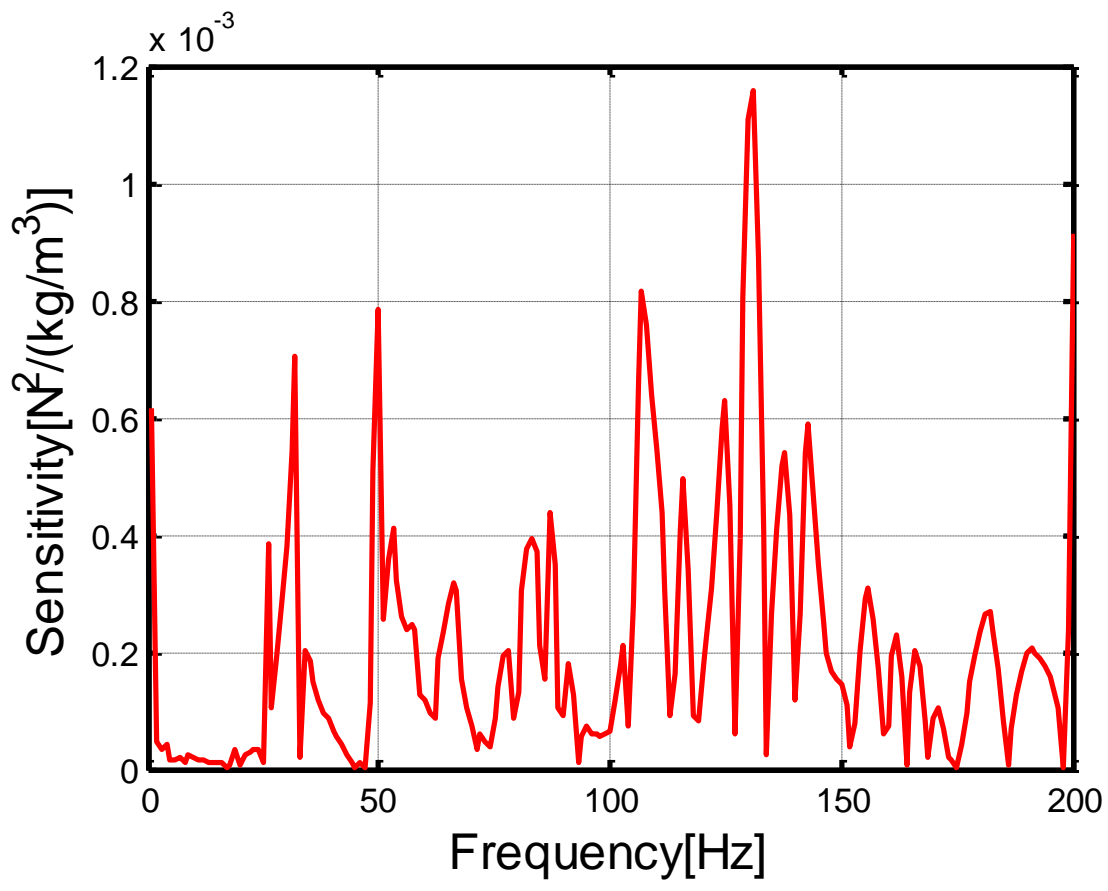

(c)

Fig. 17. (a) Sensitivity of the Auto-PSD of exciting point I to elastic modulus of exterior plate; (b) Sensitivity of the Auto-PSD of exciting point II to material density of exterior plate; (c) Sensitivity of the Auto-PSD of exciting point II to material density of inner plate. 


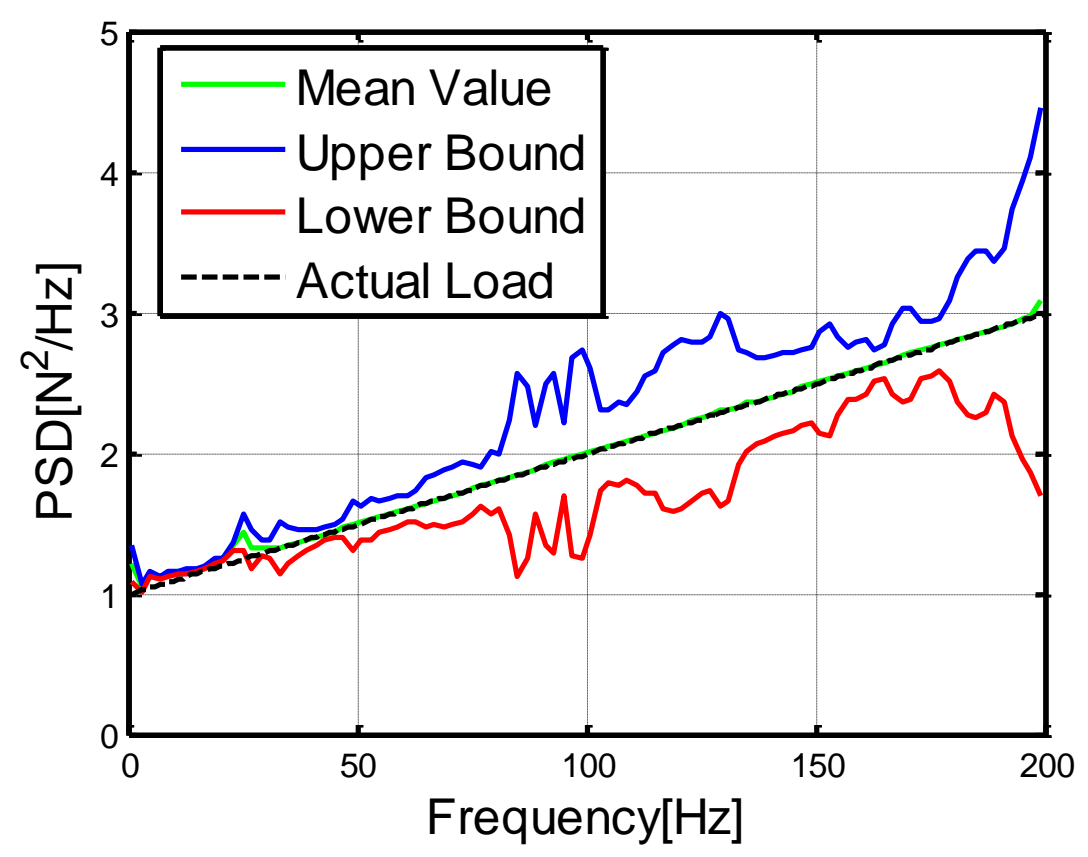

(a)

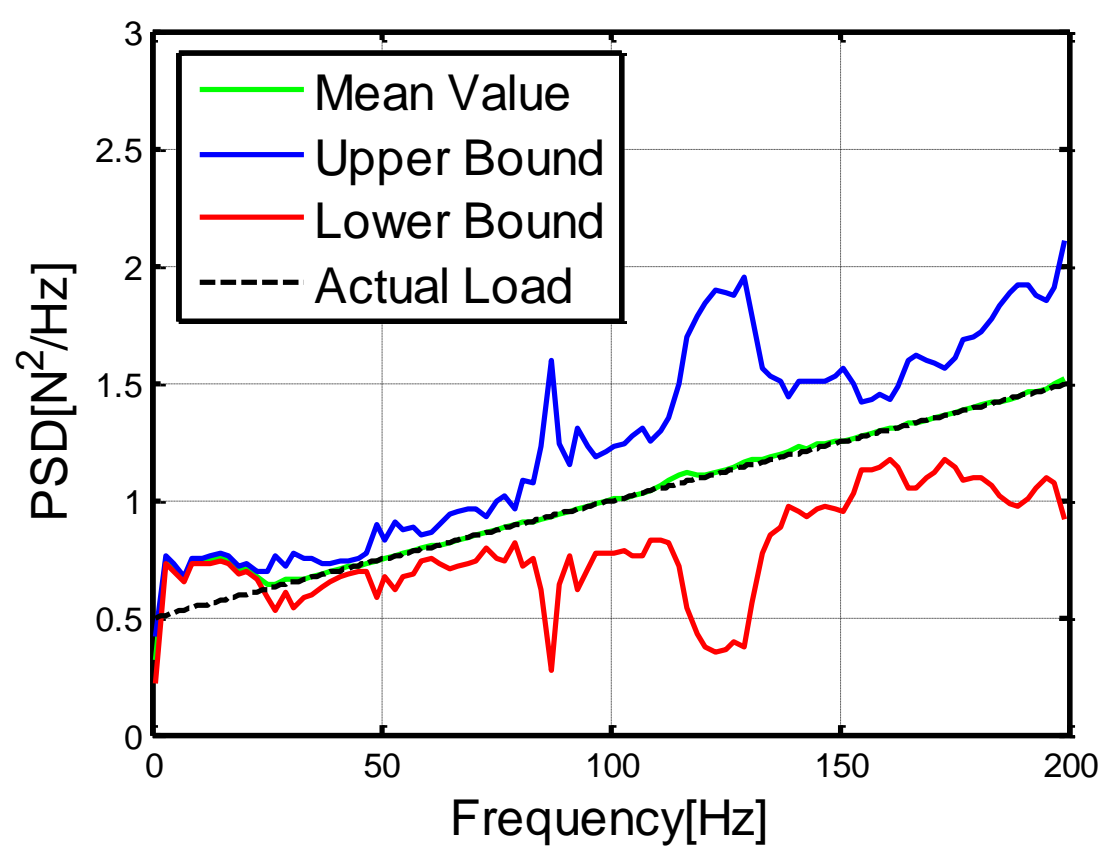

(b)

Fig. 18. The bounds of Auto-PSD of the identified random excitation (a) exciting point I; (b) exciting point II. 


\subsection{A vehicle trim body problem}

In practical engineering circumstance, a vehicle is subjected to various random dynamic loads such as road excitation and wind loading. Hence, determining the random excitations is crucial to evaluate the fatigue reliability and assess the NVH (Noise, Vibration and Harshness) performance. Owing to the restriction of engineering technology nowadays, it is generally difficult or even impractical to measure random dynamic loads directly. On the contrary, measuring the dynamic responses of the structure including dynamic strains, dynamic displacements and accelerations, is a relatively easier way which can be adopted to identify the dynamic loads indirectly. Thus, the matrix inversion is the most common method of identifying random excitation on the vehicle. In this section, to further demonstrate the effectiveness and feasibility of the modified regularization method for more complicated dynamic structures in practical engineering application, the proposed method is applied on a vehicle trim body.

The exciting points and responses points are selected in $z$-direction as shown in Fig. 19. The random dynamic acceleration responses are obtained through the given constructed random excitations, and a noise is added to the computational responses as shown in Fig. 20. The condition number of FRF matrix shown in Fig. 21 is computed over the frequency range (from $0 \mathrm{~Hz}$ to $200 \mathrm{~Hz}$ ). 


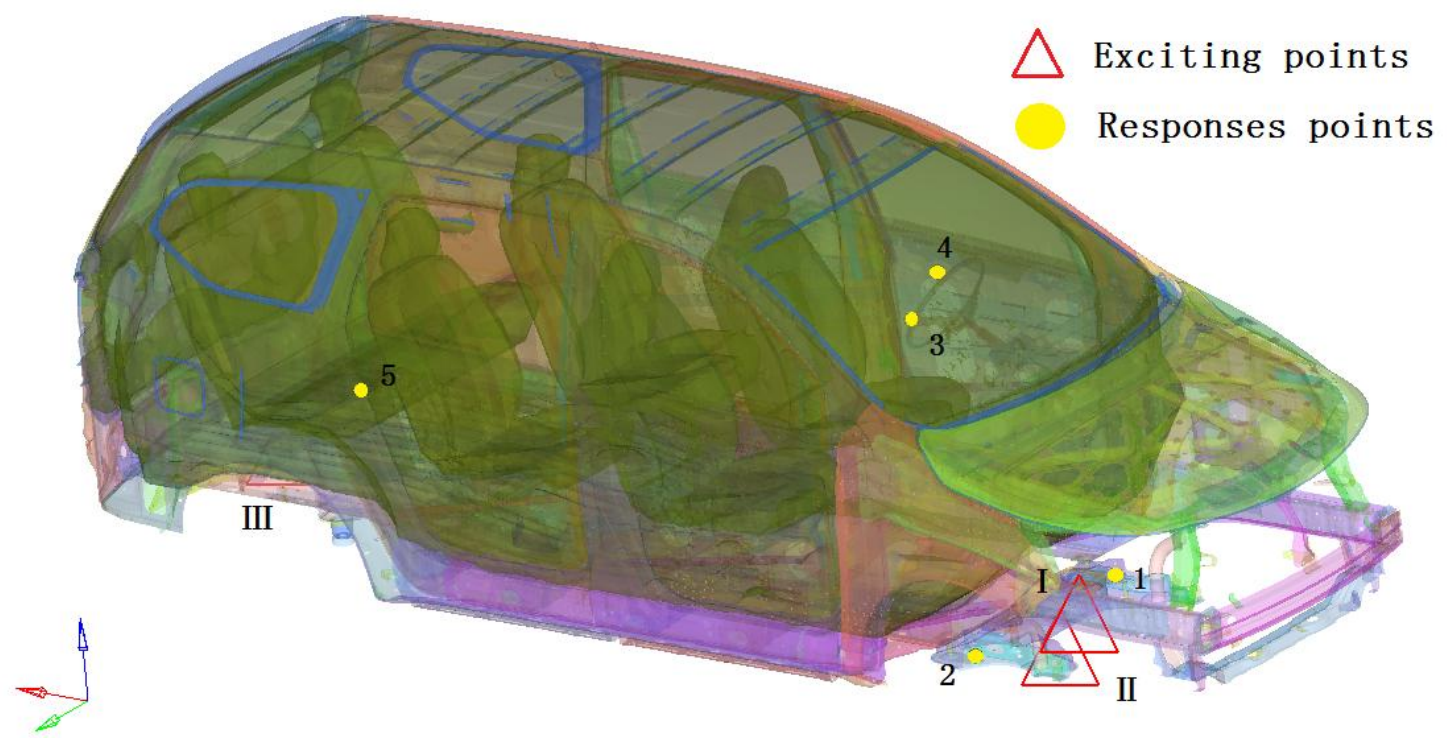

Fig. 19. The finite element model of vehicle trim body.

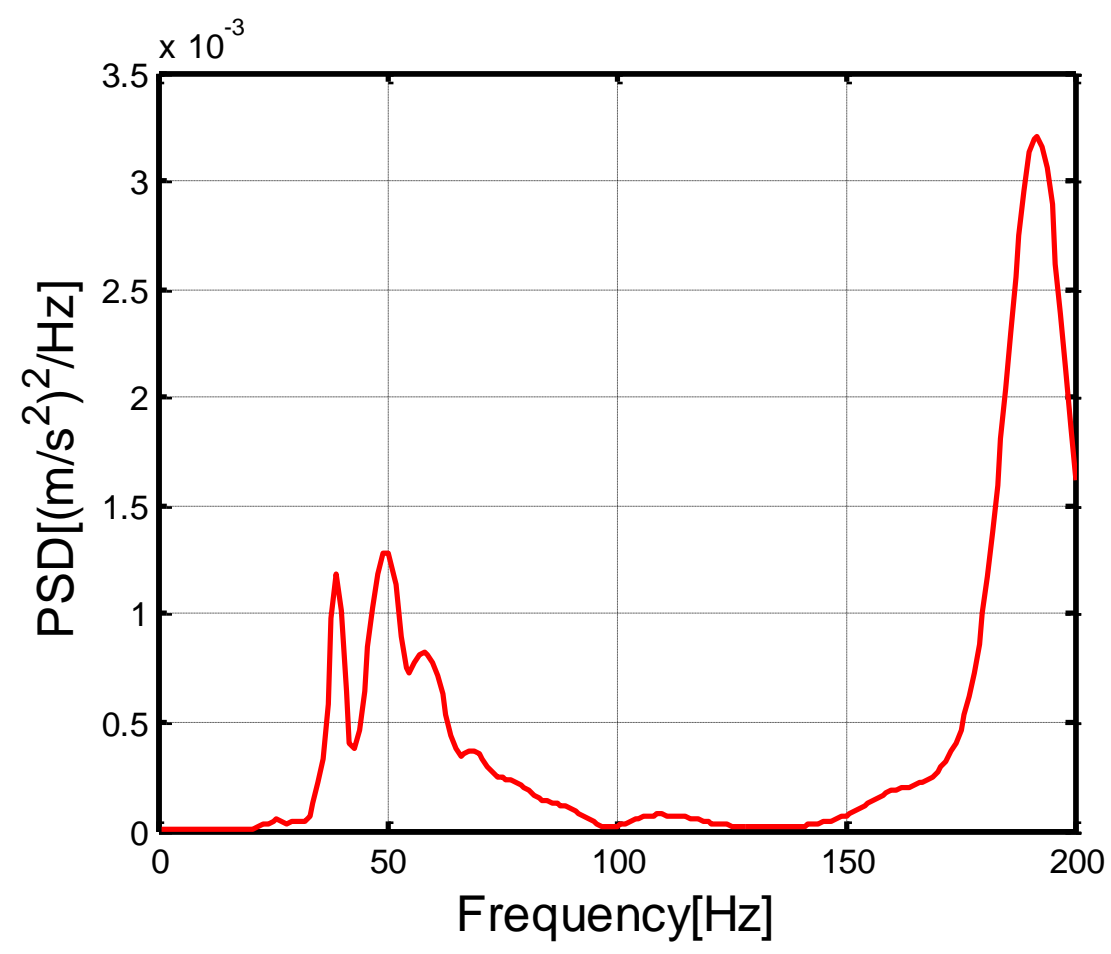

Fig. 20. Auto-PSD function of the random dynamic acceleration response of point 3 with $10 \%$ random noise. 


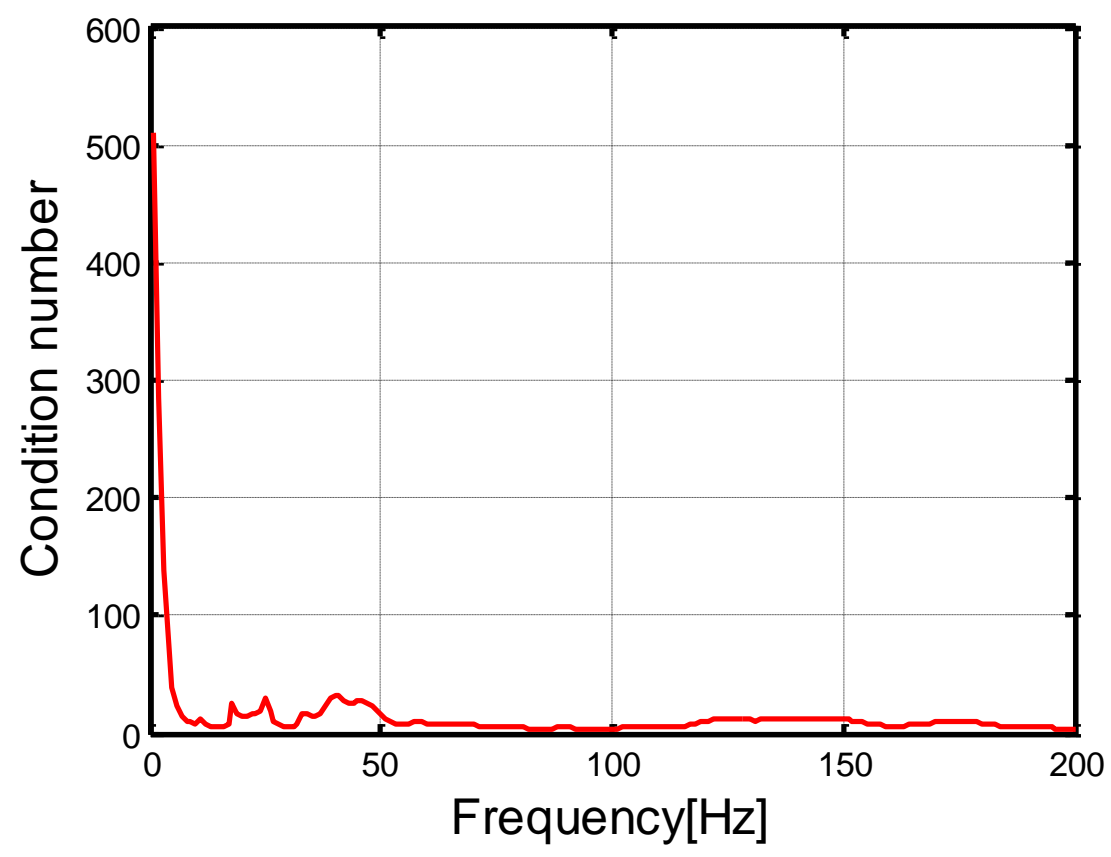

Fig. 21. Condition number of the FRF matrix.

The results of the identified loads by modified Tikhonov regularization method and other two identified methods are compared with the actual excitations as shown in Fig. 22(a-c) and Fig. 23(a-b). It can be seen that the modified regularization method is able to reduce the error remarkably and match the actual excitation fairly well in the whole frequency region. As expected, the numerical results from traditional Tikhonov regularization method are not acceptable. In addition, it is seen that the identified loads for different exciting points from the Moore-Penrose pseudo inverse method are not satisfactory in the low frequency range. Thus, the proposed modified regularization method is demonstrated again to be effective and feasible for practical engineering structures. 


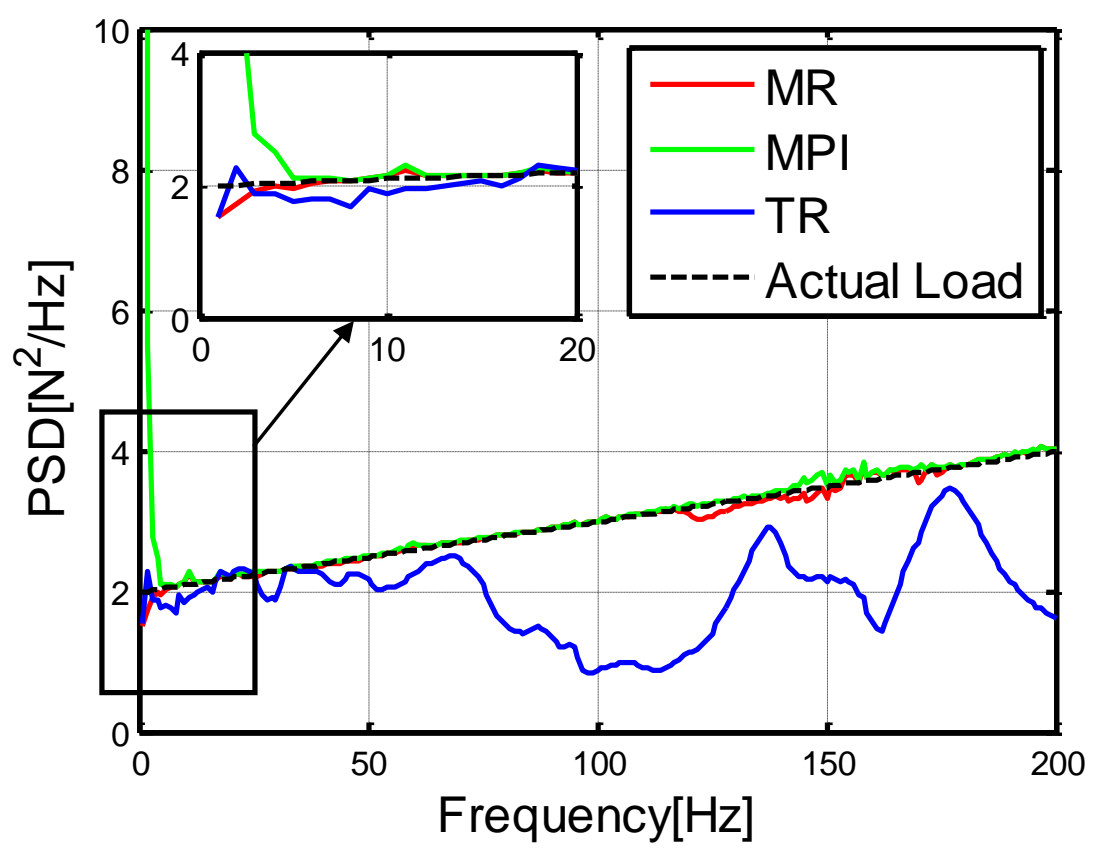

(a)

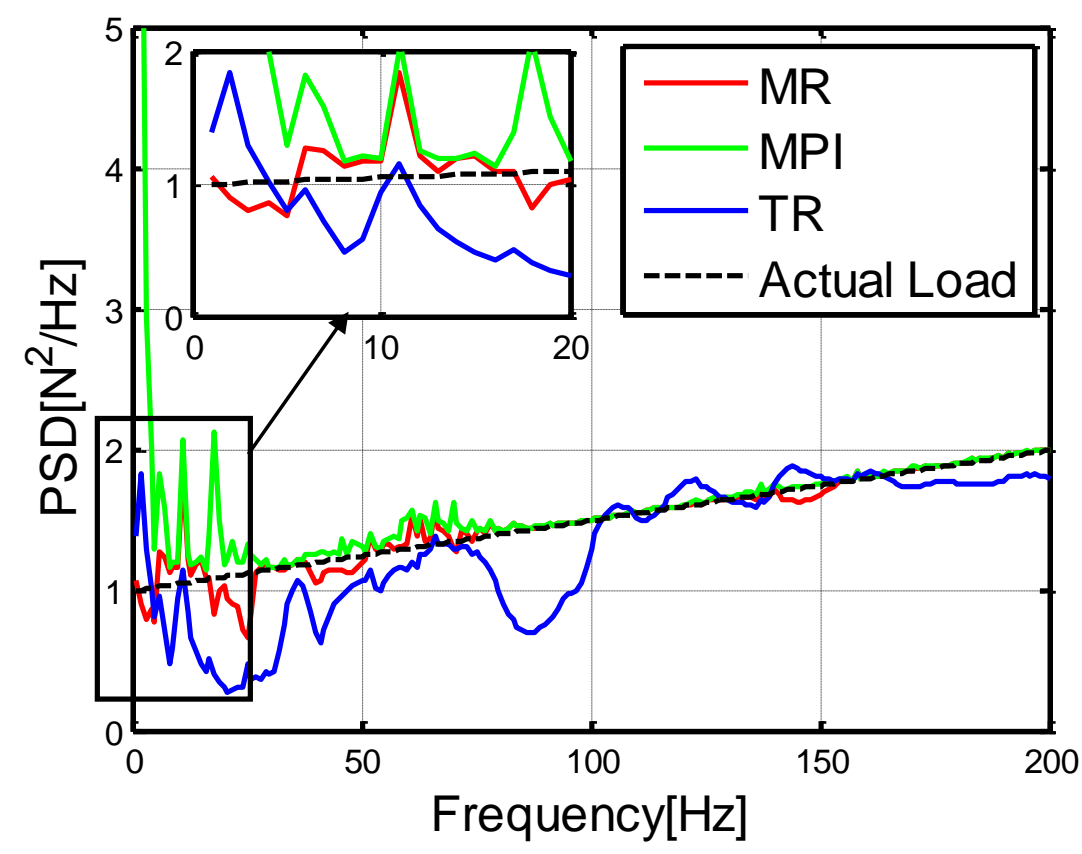

(b) 


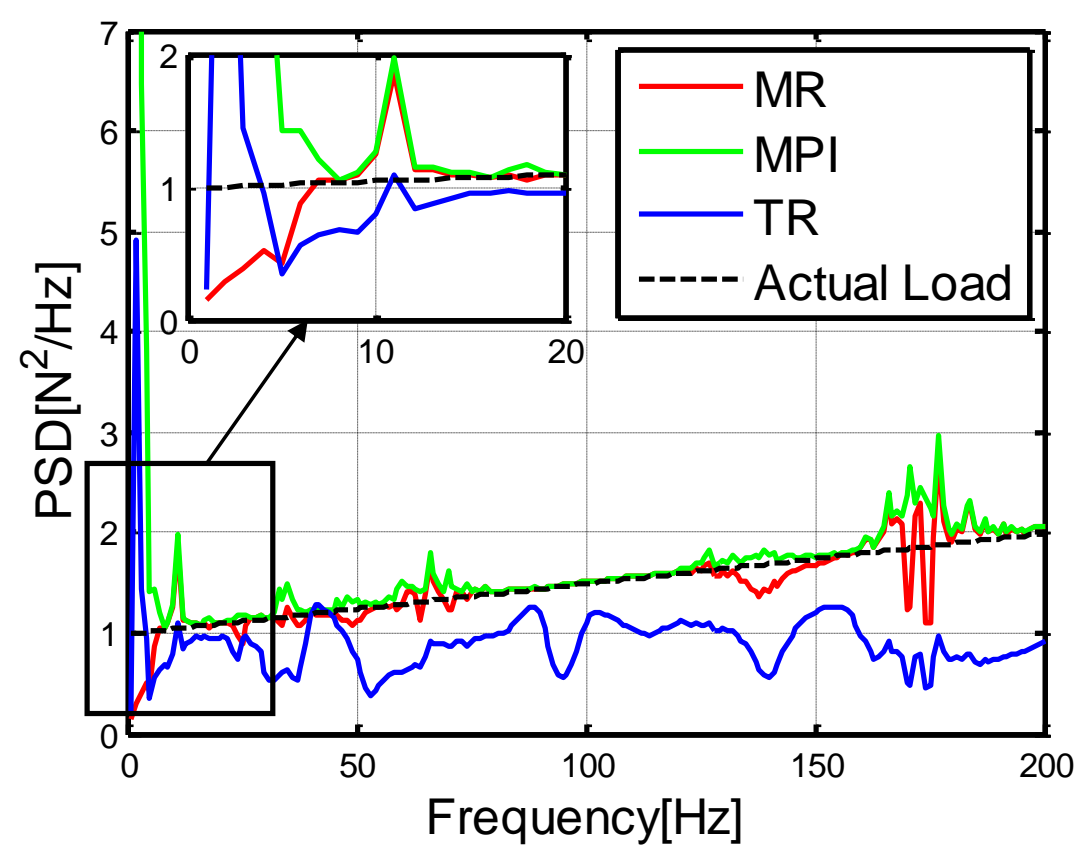

(c)

Fig. 22. Comparison of the Auto-PSD of identified load by modified regularization method and other two identified methods with actual loads: (a) exciting point I; (b) exciting point II; (c) exciting point III.

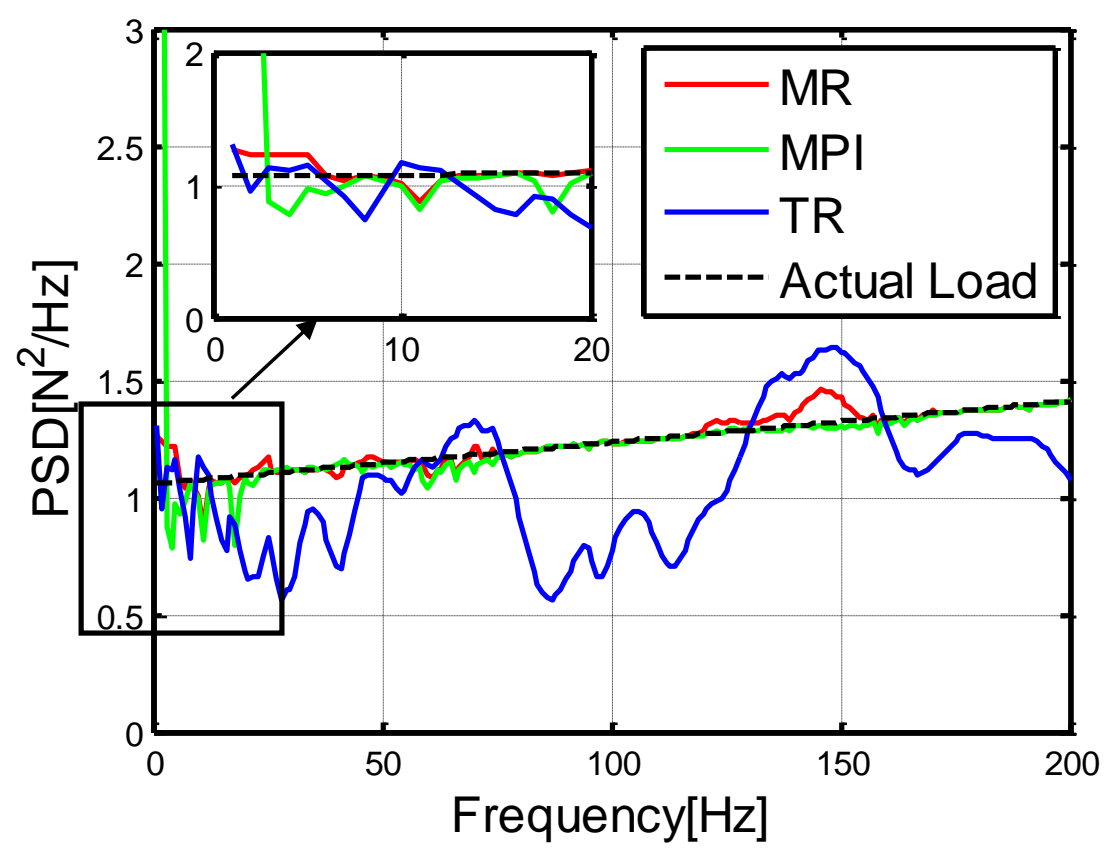

(a) 


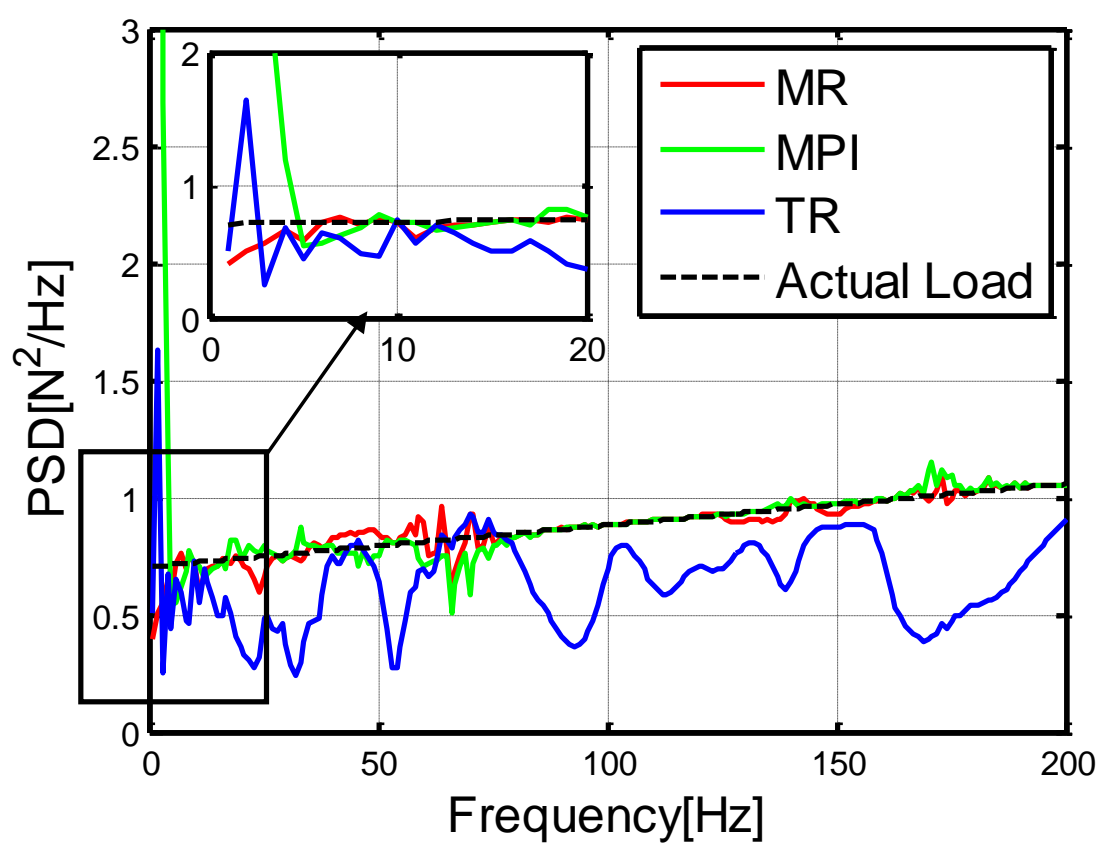

(b)

Fig. 23. Comparison of the Cross-PSD magnitude of identified load by modified regularization method and other two identified methods with actual loads: (a) between exciting point I and exciting point II; (b) between exciting point II and exciting point III.

\section{Conclusions}

In this work, the random dynamic load identification problems for stochastic structures are analyzed and investigated in detail. An inverse pseudo excitation perturbation method (IPEPM) and a modified regularization method are formulated for solving the combined random excitation identification problems. Based on numerical results, the major conclusions can be derived as follows:

a) The proposed modified regularization method is not sensitive to the selection of measurement locations in comparison with Moore-Penrose method and traditional Tikhonov regularization method. 
b) Due to the proper regularization effect, the proposed modified regularization method is very effective to solve the ill-posed problems and improve the accuracy as well as stability of identified random excitation.

c) The proposed modified regularization method is always able to identify multisource loads that match the actual ones from low to high frequency domains.

d) The matrix perturbation method with the modified regularization method is also studied on the several practical models with uncertainty, which can generate the bounds of identified loads effectively. The actual loads are mostly located within the bounds, which strongly validates that the proposed method is very effective and offers a high value of engineering practicability in the dealing with uncertain structures and forces.

\section{Acknowledgements}

The project is supported by the Science Fund of State Key Laboratory of Advanced Design and Manufacturing for Vehicle Body No. 31615002. The authors wish to thank and Research Project of State Key Laboratory of Mechanical Systems and Vibration MSV201711 and Research Project of State Key Laboratory of Structural Analysis for Industrial Equipment (Grant No. GZ 1609) for the support.

\section{References}

[1] G.R. Liu, W.B. Ma, X. Han, An inverse procedure for identification of loads on composite laminates, Composites Part B, 33 (2002) 425-432.

[2] G.R. Liu, W.B. Ma, X. Han, Inversion of loading time history using displacement response of composite laminates: Three-dimensional cases, Acta Mechanica, 157 (2002) 223-234.

[3] J. Liu, X. Sun, X. Han, C. Jiang, D. Yu, A novel computational inverse technique for load identification using the shape function method of moving least square fitting, Computers \& Structures, 144 (2014) 127-137.

[4] J. Liu, X. Meng, D. Zhang, C. Jiang, X. Han, An efficient method to reduce ill-posedness for structural dynamic load identification, Mechanical Systems \& Signal Processing, 95 (2017) 273-285.

[5] F. Naets, J. Cuadrado, W. Desmet, Stable force identification in structural dynamics using Kalman filtering 
and dummy-measurements, Mechanical Systems \& Signal Processing, s 50-51 (2015) 235-248.

[6] H. Lee, Y.S. Park, Error analysis of indirect force determination and a regularisation method to reduce force determination error, Mechanical Systems \& Signal Processing, 9 (1995) 615-633.

[7] T.P. Nordberg, I. Gustafsson, Using QR factorization and SVD to solve input estimation problems in structural dynamics, Computer Methods in Applied Mechanics \& Engineering, 195 (2006) 5891-5908.

[8] F. Presezniak, P.A.G. Zavala, G. Steenackers, K. Janssens, J.R.F. Arruda, W. Desmet, P. Guillaume, Acoustic source identification using a Generalized Weighted Inverse Beamforming technique, Mechanical Systems \& Signal Processing, 32 (2012) 349-358.

[9] E. Turco, Load distribution modelling for pin-jointed trusses by an inverse approach, Computer Methods in Applied Mechanics \& Engineering, 165 (1998) 291-306.

[10] J. Liu, X. Meng, C. Jiang, X. Han, D. Zhang, Time - domain Galerkin method for dynamic load identification, International Journal for Numerical Methods in Engineering, 105 (2016) 620-640.

[11] G.R. Liu, X. Han, Computational inverse techniques in nondestructive evaluation, CRC Press.

[12] F.D.B. Jr, W.G. Flannelly, Model verification of force determination for measuring vibratory loads, Journal of the American Helicopter Society, 24 (1979) 10-18.

[13] N. Okubo, S. Tanabe, T. Tatsuno, Identification of Forces Generated by a Machine Under Operating Condition, (1985).

[14] P.C. Hanson, Rank-deficient and discrete ill-posed problems, SIAM, 1998.

[15] J. O'Callahan, F. Piergentili, Force Estimation Using Operational Data, Proceedings of SPIE - The International Society for Optical Engineering, 2768 (1996).

[16] Y. Liu, W.S.S. Jr, Dynamic force identification based on enhanced least squares and total least-squares schemes in the frequency domain, Journal of Sound \& Vibration, 282 (2005) 37-60.

[17] S. Chakraborty, S.S. Dey, A stochastic finite element dynamic analysis of structures with uncertain parameters, International Journal of Mechanical Sciences, 40 (1998) 1071-1087.

[18] G.I. Schuëller, A state-of-the-art report on computational stochastic mechanics, Probabilistic Engineering Mechanics, 12 (1997) 197-321.

[19] J. Li, S. Liao, Response analysis of stochastic parameter structures under non-stationary random excitation, Computational Mechanics, 27 (2001) 61-68.

[20] J. Lin, W. Zhang, J. Li, Structural responses to arbitrarily coherent stationary random excitations, Computers \& Structures, 50 (1994) 629-633.

[21] W.K. Liu, T. Belytschko, A. Mani, Probabilistic finite elements for nonlinear structural dynamics, Computer Methods in Applied Mechanics \& Engineering, 56 (1986) 61-81.

[22] H.U. Köylüoğlu, S.R.K. Nielsen, A.Ş. Çakmak, Stochastic Dynamics of Nonlinear Structures with Random Properties Subject to Stationary Random Excitation, Journal of Sound \& Vibration, 190 (1994) 821-841.

[23] S. Chen, Matrix perturbation theory in structural dynamic design, Science Press, 2007.

[24] A. Chatterjee, Sensitivity and Error Analysis of a Coupled Micro-Resonator Array for Ultra-Sensitive Mass Detection Using Matrix Perturbation Theory, IEEE Sensors Journal, 16 (2016) 1304-1309.

[25] D.L. Carnì, C. Scuro, F. Lamonaca, R.S. Olivito, D. Grimaldi, Damage analysis of concrete structures by means of acoustic emissions technique, Composites Part B: Engineering.

[26] Z. He, J. Hu, E. Li, An uncertainty model of acoustic metamaterials with random parameters, Comput Mech, (2018) 1-14.

[27] Z. He, Y. Wu, E. Li, Topology optimization of structure for dynamic properties considering hybrid uncertain parameters, Struct Multidiscip O, 57 (2018) 625-638. 
[28] J. Liu, X. Sun, X. Han, C. Jiang, D. Yu, Dynamic load identification for stochastic structures based on Gegenbauer polynomial approximation and regularization method, Mechanical Systems \& Signal Processing, $\mathrm{s}$ 56-57 (2015) 35-54.

[29] J. Liu, X. Han, C. Jiang, H.M. Ning, Y.C. Bai, Dynamic load identification for uncertain structures based on interval analysis and regularization method, International Journal of Computational Methods, 8 (2011) 667-683.

[30] Z.C. He, X.Y. Lin, E. Li, A novel method for load bounds identification for uncertain structures in frequency domain, International Journal of Computational Methods, (2017) 1850051.

[31] A. Batou, C. Soize, S. Audebert, Model identification in computational stochastic dynamics using experimental modal data, Mechanical Systems \& Signal Processing, 50-51 (2015) 307-322.

[32] A. Batou, C. Soize, Experimental identification of turbulent fluid forces applied to fuel assemblies using an uncertain model and fretting-wear estimation, Mechanical Systems \& Signal Processing, 23 (2009) 2141-2153.

[33] A. Batou, C. Soize, Identification of stochastic loads applied to a non-linear dynamical system using an uncertain computational model and experimental responses, Computational Mechanics, 43 (2009) 559-571.

[34] E. Zhang, J. Antoni, P. Feissel, Bayesian force reconstruction with an uncertain model, Journal of Sound \& Vibration, 331 (2012) 798-814.

[35] Y. Jia, Z. Yang, N. Guo, L. Wang, Random dynamic load identification based on error analysis and weighted total least squares method, Journal of Sound \& Vibration, 358 (2015) 111-123.

[36] Y. Jia, Z. Yang, Q. Song, Experimental study of random dynamic loads identification based on weighted regularization method, Journal of Sound \& Vibration, 342 (2015) 113-123.

[37] J.H. Lin, X.L. Guo, H. Zhi, W.P. Howson, F.W. Williams, Computer simulation of structural random loading identification, Computers \& Structures, 79 (2001) 375-387.

[38] A.N. Thite, D.J. Thompson, Selection of response measurement locations to improve inverse force determination, Applied Acoustics, 67 (2006) 797-818.

[39] A.N. Tikhonov, V.Y. Arsenin, Solutions of Ill-posed Problems, Mathematics of Computation, 32 (1977) 491-491.

[40] A. Tveito, H.P. Langtangen, B.F. Nielsen, X. Cai, Parameter Estimation and Inverse Problems, International Geophysics, 67 (2010) 411-421.

[41] G.H. Golub, C. Reinsch, Singular Value Decomposition and Least Squares Solutions, Springer Berlin Heidelberg, 1971.

[42] P.C. Hansen, REGULARIZATION TOOLS: A Matlab package for analysis and solution of discrete ill-posed problems, Numerical Algorithms, 6 (1994) 1-35.

[43] J. Wang, S.S. Law, Q.S. Yang, Sensor placement methods for an improved force identification in state space, Mechanical Systems \& Signal Processing, 41 (2013) 254-267. 